\title{
ESSENTIAL EXTENSIONS OF PARTIAL ORDERS ON GROUPS
}

\author{
BY \\ JORGE MARTINEZ( $\left.{ }^{1}\right)$
}

\begin{abstract}
Let $(G, P)$ be an $l$-group and $\mathscr{C}(P)$ be the lattice of convex $l$-subgroups of $(G, P)$. We say that the $l$-cone $Q$ is essential over $P$ if $\mathscr{C}(Q)$ is contained in $\mathscr{C}(P)$. It is shown that for each nonzero $x$ in $G$ and each $Q$-value $D$ of $x$, there is a $P$-value $C$ of $x$ containing $D$ and no other $Q$-value of $x$. We specialize to those essential extensions for which the above $C$ always depends uniquely on $x$ and $D$; these are called very essential extensions. We show that if $(G, P)$ is a representable $l$-group then $P$ is the meet of totally ordered very essential extensions of $P$. Further we investigate connections between the existence of total very essential extensions and both representability and normal valuedness. We also study the role played by the various radicals in the theory.

The same two classes of extensions are treated in the context of abelian Riesz groups. Similar questions about existence of such total orders are dealt with. The main result in this connection is that such total extensions always exist for finite valued pseudo lattice groups, and that the original cone is the meet of them.
\end{abstract}

Introduction. Not too much has been done in the theory of partially ordered groups in the area of extensions or refinements of partial orders. Most of what is known in this field is treated in [9]. The purpose of this paper is to study some aspects of the theory of extensions. Specifically, essential extensions are treated; in $\S 1$ all is done in the context of $l$-groups, whereas in $\$ 2$ the spectrum is broadened to Riesz groups, but then only abelian Riesz groups.

An $l$-extension $Q$ of an $l$-cone $P$ is essential if $\mathscr{C}(Q) \subseteq \mathscr{C}(P) ; \mathscr{C}(P)$ denotes the set of convex $l$-subgroups of the $l$-group $(G, P)$. The Structure Lemma (1.1) seems to be the cornerstone of most of the results, but it is easy enough to prove. It says that if $Q$ is essential over $P$, then for each $x \neq 0$ and each $Q$-value $D$ of $x$ there is a $P$-value $C$ of $x$ that contains $D$ and no other $Q$-value of $x$. In this context we say that $Q$ is very essential over $P$ if said $P$-value $C$ is uniquely determined by $x$ and $D$.

Received by the editors October 6, 1969.

AMS 1968 subject classifications. Primary 0675.

$K e y$ words and phrases. $l$-extension, essential $l$-extension, very essential $l$-extension, regular subgroup, value, finite valued group, normal valued group, representable group, preservation of disjointness, Riesz group, Rz-extension, pseudo lattice group, decomposition into special elements.

(1) The author wishes to thank Professor Paul Conrad for spending the time to discuss these ideas with him, and also for giving him many valuable examples and suggestions. 
If $(G, Q)$ is a normal valued $l$-group then $Q$ is very essential over $P$ if and only if, for each $x \in Q$, the $P$-negative part of $x$ is infinitely small compared to the $P$ positive part of $x$ (relative to $Q$, of course). This result implies that in the context of vector lattices the notions of essential and very essential extensions coincide. The existence of "enough" totally ordered very essential extensions of representable groups is established, and some technical results are proved concerning essential extensions. We then examine the question of what $l$-groups admit totally ordered very essential extensions. Finally we study the connections that the various "radicals" might have in this theory, and we give an example of an l-group which is not totally ordered, but whose cone admits no proper very essential $l$-extensions (see the example (1-D)).

$\S 2$ deals with the same two notions in the context of abelian Riesz groups; indeed, most of the time we will be dealing with $p l$-groups. One obtains analogues of the Structure Lemma and Theorems (1.2) and (1.3) for extensions of $p l$-groups. Proposition (2.5) really says that there are plenty of very essential extensions around. We show that at least for finite valued $p l$-groups $(G, P)$ totally ordered very essential extensions exist, and $P$ is the meet of them (Corollary (2.8.1) and Proposition (2.11)). Theorem (2.6) presents a puzzling answer to the question of whether a very essential extension of a $p l$-cone is again a $p l$-cone. The characterization of Theorem (2.10) is really what aroused our interest in these extensions; chronologically it came first. Finally we devote some space to the question of the existence of minimal very essential $l$-extensions of $p l$-cones.

For the basic theory of $l$-groups and vector lattices we refer the reader to [9]. In $\S 1$ the material in [1], [4], [5], [6] and [8] might be particularly helpful. For $§ 2$ familiarity with [7] and perhaps also [10], [3] and [12] is strongly recommended. Since the theory presented here is essentially self-contained, the references can be helpful only in providing an adequate background.

Some notation and terminology. The letters $\boldsymbol{Z}, \boldsymbol{Q}$, and $\boldsymbol{R}$ will denote the set of integers, rationals and real numbers respectively. If $(G, P)$ is an $l$-group, call a convex $l$-subgroup $D$ regular if it is maximal without some element $x \neq 0$. We then say that $D$ is a value of $x$. The intersection of all convex $l$-subgroups that properly contain $D$ covers $D$; we will denote it throughout by $\bar{D}$. The set of right cosets of $\bar{D}$ modulo $D$ is then totally ordered in the order induced from $P$. If $D$ is normal in $\bar{D}$ then $\bar{D} / D$ is o-isomorphic to a subgroup of the reals $R$. A convex $l$-subgroup $B$ is prime if $a \wedge b=0$ implies that $a \in B$ or $b \in B$. We remark that all regular subgroups are prime. An $l$-group $(G, P)$ is finite valued if every nonzero element $x$ has only a finite number of values. An element $a$ is special at the regular subgroup $D$ if $D$ is its only value. (See [6, §3].)

By a root system we mean a partially ordered set in which no two incomparable elements have a common lower bound. The set of all regular subgroups of an l-group $(G, P)$, which we will denote by $\mathscr{R}(P)$, is a root system [6, §3]. Let $\Lambda$ be a partially ordered set and $H_{\lambda}$ be an o-group for each $\lambda \in \Lambda$. Let $\Pi=\Pi\left(\Lambda, H_{\lambda}\right)$ be 
the product of the $H_{\lambda}$ over $\Lambda$ and $V=V\left(\Lambda, H_{\lambda}\right)$ be the subgroup of $\Pi$ consisting of all elements whose supports have no infinite ascending sequences relative to $\Lambda$. We can define a partial order on $V$ by taking for its positive cone the set $\{v \in V$ : every maximal nonzero component is positive $\}$. If $\Lambda$ is a root system then $V$ is an l-group with this order [2, §2]. In any case $V$ is a pl-group [7, §4]. $\Sigma=\Sigma\left(\Lambda, H_{\lambda}\right)$ will denote the small direct sum. We shall use the symbol $\boxplus$ to indicate cardinal direct sum, i.e. direct sum of $l$-groups with the "pointwise" order. If $G$ is a group and $S$ is any subset, then $\langle S\rangle$ denotes the subgroup generated by $S$.

In the context of $\$ 2$, the terms "value", "regular subgroup", "finite valued", etc., mean the same thing as for $l$-groups, but they must be taken relative to the set of o-ideals of the p.o. group. (An o-ideal is a directed, convex subgroup.)

Throughout the text "vector lattice" will mean "real vector lattice."

1. Essential $l$-extensions of $l$-groups. Throughout this section $(G, P)$ will denote an $l$-group $G$ with its positive cone $P$. By an l-extension $Q$ of $P$ we shall mean a cone $Q \supseteq P$, such that $(G, Q)$ is again an l-group. $Q$ is an essential $l$ extension of $P$ if $\mathscr{C}(Q) \subseteq \mathscr{C}(P)$.

Let $Q$ be an $l$-extension of the lattice cone $P$ (abbr. $l$-cone). Let $D \in \mathscr{C}(Q)$; then $D \cap P$ is a convex subsemigroup of $(G, P)$ that contains 0 , so we know that $\langle D \cap P\rangle \in \mathscr{C}(P)$. Recall that $\langle D \cap P\rangle=\{x-y: x, y \in D \cap P\}$ and $\langle D \cap P\rangle^{+}$ $=D \cap P$ (see [4, p. 172]). Define $D \tau=\langle D \cap P\rangle$; then $D \tau \cap P=D \cap P$, and $D \tau$ is the largest convex $l$-subgroup of $(G, P)$ contained in $D$. It is clear that $Q$ is essential over $P$ if and only if $D \tau=D$, for all $D \in \mathscr{C}(Q)$.

We note the following properties of essential extensions: let $P, Q$ and $Q^{\prime}$ be l-cones for $G$ such that $P \subseteq Q \subseteq Q^{\prime}$;

(i) if $Q^{\prime}$ is essential over $Q$ and $Q$ is essential over $P$, then $Q^{\prime}$ is essential over $P$.

(ii) If $Q^{\prime}$ is essential over $P$ then it is also essential over $Q$.

The proof of (i) is trivial; (ii) follows immediately once we observe the following: with $P, Q$ and $Q^{\prime}$ as before, let $\tau: \mathscr{C}\left(Q^{\prime}\right) \rightarrow \mathscr{C}(P)$ be defined by $C \tau=\langle C \cap P\rangle$, for all $C \in \mathscr{C}\left(Q^{\prime}\right)$; let $\tau^{\prime}: \mathscr{C}\left(Q^{\prime}\right) \rightarrow \mathscr{C}(Q)$ and $\tau^{\prime \prime}: \mathscr{C}(Q) \rightarrow \mathscr{C}(P)$ be defined analogously. Then for all $C \in \mathscr{C}\left(Q^{\prime}\right)$, we have

$$
\begin{aligned}
C \tau^{\prime} \tau^{\prime \prime} \cap P & =C \tau^{\prime} \cap P=C \tau^{\prime} \cap Q \cap P \\
& =C \cap Q \cap P=C \cap P=C \tau \cap P
\end{aligned}
$$

hence $C \tau^{\prime} \tau^{\prime \prime}=C \tau$ and so $\tau^{\prime} \tau^{\prime \prime}=\tau$.

Notice that nothing is said in the preceding about $Q$ over $P$ when $Q^{\prime}$ is essential over $P$. Consider in fact the following example:

(1-A) Let $G=R^{2}, P=\{(x, y): x \geqq 0$ and $y \geqq 0\}, Q^{\prime}=\{(x, y): x>0$, or $x=0$ and $y \geqq 0\}$ and $Q=\{(x, y): x \geqq 0$ and $y \geqq-x\}$. Then $(G, P),(G, Q)$ and $\left(G, Q^{\prime}\right)$ are all vector lattices $\left(\left(G, Q^{\prime}\right)\right.$ is totally ordered). It is clear that $Q^{\prime}$ is an essential extension of $P$, and hence of $Q$. However, the subgroup $D=\{(x,-x): x \in \boldsymbol{R}\}$, while being a convex o-subgroup of $(G, Q)$, is not in $\mathscr{C}(P)$, for $D \cap P=0$. 
We shall have occasion to use this example again later. The following lemma is easy to prove, but very useful in the sequel.

(1.1) Structure Lemma. Let $Q$ be an essential l-extension of $P, g \neq 0$ and $D$ be a $Q$-value of $g$. (By a $Q$-value of $g$ we mean, of course, a value relative to the order $Q$.) Then $D$ is contained in a P-value $C$ of $g$, and no other $Q$-value of $g$ is contained in $C$.

Proof. First $D \in \mathscr{C}(Q) \subseteq \mathscr{C}(P)$ and $g \notin D$, so by Zorn's Lemma $D$ is contained in a $P$-value $C$ of $g$. Suppose $D^{\prime}$ is a $Q$-value of $g$ which is also contained in $C$. Let $K$ be the subgroup generated by $D$ and $D^{\prime}$. Then, since $D$ and $D^{\prime}$ are in $\mathscr{C}(P), K$ is the smallest convex $l$-subgroup of $(G, P)$ containing both $D$ and $D^{\prime}$; this says that $K \subseteq C$, and hence that $g \notin K$. On the other hand $K \in \mathscr{C}(Q)$, and so by the maximality of $D$ and $D^{\prime}$ relative to $Q$ it follows that $D=K=D^{\prime}$.

(1.1.1) Corollary. Let $Q$ be an essential l-extension of $P$.

(i) If $g \neq 0$ is special relative to $P$ (henceforth, $P$-special) it is also $Q$-special.

(ii) If $(G, P)$ is finite valued, so is $(G, Q)$.

We remark in passing that it is unknown whether any l-extension of a finite valued $l$-cone $P$ is again finite valued.

Let $(G, P)$ be an l-group; if $x, y \in P$ and $m x \geqq y$ and $n y \geqq x$ for some positive integers $m$ and $n$, we say $x$ and $y$ are Archimedean equivalent; we shall denote this occurrence by $x \sim y$.

(1.2) Theorem. Let $Q$ be an l-extension of $P$; $Q$ is essential over $P$ if and only if, for every $x \in Q, x \sim x \vee_{P} 0$, relative to $Q$.

Proof. Let us write $x_{P}^{+}$for $x \vee_{P} 0$.

Suppose first that $x \sim x_{P}^{+}$, for all $x \in Q$. If $D \in \mathscr{C}(Q)$ and $a \in D \cap Q$, then $a_{P}^{+} \in D$, since $a \sim a_{P}^{+}$(rel. $Q$ ). But then $a_{P}^{-}=a_{P}^{+}-a \in D$. This suffices to prove that every element of $D$ is the difference of two elements of $P$. It follows then that $D$ is $P$-directed, and since it was $Q$-convex it is certainly $P$-convex. Thus $D$ is a $P$ convex $l$-subgroup, and so $Q$ is essential over $P$.

Conversely, suppose $Q$ is essential over $P$, and let $x \in Q$. Let $K$ be the convex l-subgroup of $(G, Q)$ generated by $x$. Then $K \in \mathscr{C}(P)$ and therefore $x_{P}^{+} \in K$, which implies that $x_{P}^{+} \leqq n x$ (rel. $Q$ ), for a suitable positive integer $n$. Since $x_{P}^{+}$already exceeds $x$ (rel. $P$ ) we conclude that $x_{P}^{+} \sim x$, and the proof is complete.

With regard to the Structure Lemma we point out that it may turn out that if $Q$ is essential over $P$, and $D$ is a $Q$-value of an element $x$, then there are two or more $P$-values of $x$ containing $D$. The lemma just gives the existence of at least one such $P$-value. Consider for example the following:

(1-B) Let $G=\{\alpha+\beta \pi: \alpha, \beta \in Q\}, P=\{\alpha+\beta \pi: \alpha, \beta \geqq 0\}$ and $Q$ be the usual order on $G$, the one it inherits from $\boldsymbol{R} .(G, Q)$ is an Archimedean o-group and therefore $l$-simple. Thus $Q$ is clearly an essential extension of $P$. But if we let $x=1+\pi$, then 0 is the only $Q$-value of $x$, while $Q$ and $Q \pi$ are its two $P$-values. 
Therefore if $Q$ is an essential extension of $P$, and for each $g \neq 0$ and each $Q$-value $D$ of $g$ there is exactly one $P$-value $C$ of $g$ containing $D$, we will say that $Q$ is a very essential extension of $P$. (Example (1-B) is an essential extension which is not very essential.) The following theorem gives (in a corollary) an elementwise characterization of very essential extensions.

(1.3) Theorem. Let $(G, P)$ be an l-group, and $Q$ be an essential l-extension of $P$. Then $Q$ is very essential over $P$ if and only if for each $x \in Q \mid 0$ and each $Q$-value $D$ of $x$ we have $D+x_{P}^{+}=D+x$.

Proof. (Sufficiency) We must show that if $D$ is a $Q$-value of the nonzero element $g$, then there is exactly one $P$-value of $g$ containing $D$. It suffices to take $g \in Q$. So suppose there are two distinct $P$-values of $g$, say $C_{1}$ and $C_{2}$, that contain $D$. We claim these are both $P$-positive values of $g$. For $D+g=D+g_{P}^{+}$, so $g_{P}^{-} \in D$ and hence is in both $C_{1}$ and $C_{2}$. But then $C_{i}+g=C_{i}+g_{P}^{+}>C_{i}$ (rel. $P$ ), for $i=1,2$.

Now we may choose $a$ and $b$ as follows: $a \in \bar{C}_{1} \mid C_{1}, a \in C_{2}$, and $b \in \bar{C}_{2} \mid C_{2}, b \in C_{1}$. Namely let $\hat{a} \in C_{2} \mid C_{1}$ and $\hat{b} \in C_{1} \mid C_{2}$, with $\hat{a}, \hat{b} \in P$; then let $a=\hat{a} \wedge_{P} g_{P}^{+}$and $b=\hat{b} \wedge_{P} g_{P}^{+}$. Since $g_{P}^{+}$is in $\bar{C}_{1} \cap \bar{C}_{2}$ we have immediately that $a \in \bar{C}_{1}$ and $b \in \bar{C}_{2}$; it is also clear that $a \in C_{2}$ and $b \in C_{1}$. In view of the fact that $C_{1}$ is a prime subgroup of $(G, P)$ we deduce that $a \notin C_{1}$; similarly $b \notin C_{2}$.

But then $D$ is a $Q$-value of $a-b$; certainly $a-b \notin D$, for otherwise $a-b \in C_{1}$, and hence $a \in C_{1}$; this is of course impossible. But on the other hand $\bar{D} \in \mathscr{C}(Q)$ $\subseteq \mathscr{C}(P)$ and $g \in \bar{D}$, which implies that $a$ and $b$ are both in $\bar{D}$. We may assume without loss of generality that $D+a-b>D$ (rel. $Q$ ); then there exists a $z \in D$ such that $z+a-b>0$ (rel. $Q$ ). Obviously $C_{1}$ and $C_{2}$ are $P$-values of $z+a-b$, and by the above we ought to have $C_{i}+z+a-b>C_{i}$ (rel. $P$ ), for $i=1,2$. However, since $z, a \in C_{2}$,

$$
C_{2}+z+a-b=C_{2}-b<C_{2},
$$

a contradiction. Thus our assumption that $C_{1}$ and $C_{2}$ are distinct leads to an absurdity; we must therefore conclude that $Q$ is indeed very essential over $P$.

(Necessity) If $Q$ is a very essential extension of $P$, then by Theorem (1.2) we have that $x \sim x_{P}^{+}$(rel. $Q$ ), for all $x \in Q$. This means that $x$ and $x_{P}^{+}$have the same $Q$-values. We are to show that $x_{P}^{-} \in D$ for every $Q$-value $D$ of $x$. Suppose, by way of contradiction, that $M$ is a $Q$-value of $x$ not containing $x_{P}^{-}$; then by the Structure Lemma $M$ is contained in a $P$-value $C^{-}$of $x_{P}^{-}$. But $M$ is also a $Q$-value of $x_{P}^{+}$, and so $M$ is also contained in a $P$-value $C^{+}$of $x_{P}^{+}$. However, $C^{+}$and $C^{-}$are then distinct $P$-values of $x$, contradicting the fact that $Q$ is very essential over $P$.

This completes the proof of the theorem.

Recall that an $l$-group $(G, P)$ is said to be normal valued if every regular subgroup is normal in its cover. If $a$ and $b$ are positive in $P$ and $n a<b$, for all $n=1,2, \ldots$, we say that $a$ is infinitely small compared to $b$, and write $a \ll b$. Again suppose $a$ and $b$ are in $P$; if every value of $a$ is properly contained in some value of $b$, then $a \ll b$. 
If $(G, P)$ is also normal valued then the converse is true; that is, $a \ll b$ implies that every value of $a$ is properly contained in a value of $b$. We therefore get the following:

(1.3.1) Corollary. If $Q$ is a very essential extension of $P$ then $x_{P}^{-} \ll x_{P}^{+}$ (rel. $Q)$, for all $x \in Q$. Conversely, if $Q$ is an l-extension of $P,(G, Q)$ is normal valued and $x_{P}^{-} \ll x_{P}^{+}$for all $x \in Q$, then $Q$ is very essential over $P$.

Proof. Since $x$ is $Q$-positive, $x_{P}^{+}$exceeds $x_{P}^{-}$, and so every value of the latter is contained in a value of the former, relative to $Q$. Certainly $x$ and $x_{P}^{+}$have the same $Q$-values, and according to Theorem (1.3) $x_{P}^{-}$is in every $Q$-value of $x$. This, together with our remarks preceding the corollary, gives the desired conclusion.

Suppose, conversely, that $(G, Q)$ is normal valued, and for each $x \in Q, x_{P}^{-} \ll x_{P}^{+}$. If $x \in Q$ then

$$
2 x-x_{P}^{+}=2 x_{P}^{+}-2 x_{P}^{-}-x_{P}^{+}=x_{P}^{+}-2 x_{P}^{-} \in Q,
$$

and therefore $x \sim x_{P}^{+}$, or in other words $Q$ is essential over $P$. Further, since $x_{P}^{-} \ll x_{P}^{+}$, we have that every $Q$-value of $x_{P}^{-}$is properly contained in some $Q$-value of $x_{P}^{+}$, and so $x_{P}^{-}$is contained in every $Q$-value of $x$. By (1.3) it follows that $Q$ is very essential over $P$.

(1.3.2) Corollary. Let $(G, P)$ be a vector lattice. If $Q$ is an essential extension of $P$ such that $(G, Q)$ is again a vector lattice, then $Q$ is very essential over $P$. Conversely, if $Q$ is a very essential l-extension of $P$ then $(G, Q)$ is a vector lattice.

Proof. Suppose $(G, Q)$ is a vector lattice and $Q$ is essential over $P$. It suffices by Theorem (1.3) to show that, for each $x \in Q$ and each $Q$-value $D$ of $x, x_{P}^{-} \in D$. If $x_{P}^{-} \notin D$ then $D$ is a $Q$-value of $x_{P}^{-}$; therefore, since $\bar{D} / D \cong \boldsymbol{R}$, we have that $D+x_{P}^{-}=D+r x$, for some $0<r \in R$. Thus $r x-x_{P}^{-}=r x_{P}^{+}-(r+1) x_{P}^{-} \in D$, and if $C$ is a $P$-value of $x$ containing $D$, we get $r x_{P}^{+}-(r+1) x_{P}^{-} \in C$. Since $C$ is $P$-prime and $r x_{P}^{+} \wedge_{P}(r+1) x_{P}^{-}=0$, it follows that either $r x_{P}^{+}$or $(r+1) x_{P}^{-}$are in $C$, and hence that they both are. Since $C$ is a subspace we get that $x_{P}^{+}$and $x_{P}^{-}$are both in $C$, and thence $x \in C$, a contradiction.

Now suppose $Q$ is a very essential $l$-extension of $P$; let $x \in Q$ and $0<r \in \boldsymbol{R}$. Since $Q$ is essential over $P$, every $l$-ideal of $(G, Q)$ is an $l$-ideal of $(G, P)$ and consequently a vector subspace. Thus if $D$ is an $l$-ideal of $(G, Q), x \in D$ if and only if $t x \in D$, for $t \neq 0$ in $R$; in particular $x$ and $t x$ have the same $Q$-values. So let $K$ be a $Q$-value of $r x$; by (1.3) $K+x=K+x_{P}^{+}$, and since $\bar{K} / K$ is a real space, we have that $K+r x=K+r x_{P}^{+}>K$ (rel. $P$ ). Thus every $Q$-value of $r x$ is positive, and hence $r x \in Q$. This shows that $(G, Q)$ is a vector lattice, and the proof is complete.

(1.3.3) Corollary. Let $(G, P)$ be an l-group, $Q$ be an l-extension of $P$ such that $(G, Q)$ is normal valued. The following are all equivalent:

(1) $Q$ is very essential over $P$;

(2) for all $x, y \in P$ with $x \wedge_{P} y=0$ it follows that $x \wedge_{Q} y \ll x \vee_{Q} y$ (rel. $Q$ );

(3) for all $x, y \in P$ with $x \wedge_{P} y=0$ it follows that $x \wedge_{Q} y \ll x+y$ (rel. $\left.Q\right)$; 
(4) for all $x, y \in P$ with $x \wedge_{P} y=0$ and $y<x$ (rel. $\left.Q\right)$ it follows that $y \ll x($ rel. $Q)$;

(5) for all $x \in Q(n+1) x \geqq n x_{P}^{+}$(rel. $\left.Q\right)$, for all $n=1,2,3, \ldots$

Proof. The equivalence of (4) and (5) is trivial, and the proof is left as an exercise to the reader. (4) $\rightarrow(1)$ is half of Corollary (1.3.1). (2) $\rightarrow(3)$ is clear since $x+y$ $\geqq x \vee_{Q} y$, with $x, y \in P \subseteq Q$. (3) $\rightarrow(4)$ : if $y<x$ (rel. $Q$ ) then $y \ll x$, because $y \ll x+y$.

Finally we show that (1) implies (2); normal valuedness will not be used here! It suffices to show that $x \wedge_{Q} y$ is contained in every $Q$-value of $x \vee_{Q} y$ (with $x, y \in P$ and $x \wedge_{P} y=0$ ). Let $K$ be a $Q$-value of $x \vee_{Q} y$; then either $x \notin K$ or $y \notin K$, and so $K$ is contained in a $Q$-value of $x$ or $y$, call it $D$. $D$ cannot be a value of both $x$ and $y$, if so it would be contained in $P$-values $C_{x}$ and $C_{y}$ of $x$ and $y$ respectively. But $x \wedge_{P} y=0$, which implies that $y \in C_{x}$ and $x \in C_{y}$; therefore $C_{x}$ and $C_{y}$ are $P$-values of $x+y$, and $D$ is obviously a $Q$-value of $x+y$. This is impossible since $Q$ is very essential over $P$, and so we conclude that $D$ is not a value of both $x$ and $y$. We may in fact assume that $D$ is a $Q$-value of $x$ and $y \in D$. This says that $D$ is also a $Q$-value of $x \vee_{Q} y$, consequently $D=K$. But since $y \in D, x \wedge_{Q} y \in D$, and we have proved what we wanted.

(1.3.4) Corollary. Let $(G, P)$ be an l-group, $Q$ be a very essential l-extension of $P$ and $Q^{\prime}$ be a very essential l-extension of $Q$. Suppose in addition that $\left(G, Q^{\prime}\right)$ is normal valued; then $Q^{\prime}$ is very essential over $P$.

Proof. Let $x, y \in P$ with $x \wedge_{P} y=0$ and $x>y$ (rel. $Q^{\prime}$ ); by (2) in Corollary (1.3.3) we have that $x \wedge_{Q} y \ll x+y$ (rel. $Q$ ). Now let $x^{\prime}=x-x \wedge_{Q} y$ and $y^{\prime}=y-x \wedge_{Q} y$; then $x^{\prime}$ and $y^{\prime}$ are $Q$-disjoint, and $x^{\prime}>y^{\prime}$ (rel. $Q^{\prime}$ ). This implies that $x^{\prime} \gg y^{\prime}$ (rel. $Q^{\prime}$ ). So for all $n=2,3, \ldots\left({ }^{2}\right)$,

$$
2 x-(n-1) y=(x+y)-(n-1)\left(x \wedge_{Q} y\right)+x^{\prime}-n y^{\prime}>0,
$$

relative to $Q^{\prime}$. In particular, for $n=2 m+1$ we get that $2 x-2 m y \in Q^{\prime}$, proving that $x \gg y\left(\right.$ rel. $\left.Q^{\prime}\right)$.

(1.4) TheOREM. Let $(G, P)$ be a finite valued l-group, and $Q$ be an l-extension of $P$. Then $Q$ is very essential over $P$ if and only if the two following conditions hold:

(i) Every $P$-special element is $Q$-special.

(ii) If $x$ and $y$ are $P$-disjoint, $P$-special elements which are not $Q$-disjoint, then either $y \ll x$ or $x \ll y($ rel. $Q)$.

Proof. First suppose that $Q$ is very essential over $P$. We already know that (i) holds (Corollary (1.1.1)); to check (ii), suppose $x$ and $y$ are $P$-disjoint, $P$-special elements of $G$ which are not $Q$-disjoint. The $Q$-value of $x$ must then be comparable to the $Q$-value of $y$. But $(G, Q)$ is again finite valued and hence normal valued. We may therefore assume, without any loss of generality, that $n x>y$, for a suitable

$\left(^{2}\right)$ It might be useful to remark that in an $l$-group two elements commute with their meet and join if they also commute with each other. 
positive integer. However, $n x$ and $y$ are $P$-disjoint, and so $n x \gg y$ (rel. $Q$ ), which in turn implies that $x \gg y$.

Now assume that (i) and (ii) hold for the $l$-extension $Q$ of $P$. We show that $(G, Q)$ is finite valued. Suppose $a, b \in P$ with $a \wedge_{P} b=0$ and $a>b$ (rel. $Q$ ). Let $a=a_{1}+\cdots+a_{m}$ and $b=b_{1}+\cdots+b_{n}$ be the decompositions of $a$ and $b$ into their respective $P$-special components (see [6, Theorem 3.7]). Let $D$ be a $Q$-value of $a$; since $a \in \bar{D}$ we have by convexity that each $a_{i}$ is in $\bar{D}$. On the other hand $a \notin D$, and so some $a_{i} \notin D$; thus $D$ is the $Q$-value of some $a_{i}$ and hence, by (ii), of exactly one $a_{i}$. (Remark: it is crucial here that $D$ is the value of a special element; according to [6, Theorem 3.6], $D$ must be normal in its cover.) Since each $a_{i}$ is $Q$-special this shows that $a$ is finite valued in $Q$; similarly $b$ is finite valued in $Q$. But this is enough to show that $(G, Q)$ is finite valued; for if $M$ is a $Q$-value of the nonzero element $g$, then $M$ is contained in the $Q$-value of a $P$-special component $g_{i}$ of $g$, say $M_{i}$, and by (ii) we can choose $M_{i}$ in such a way that every other component of $g$ is in $M_{i}$. That implies that $M_{i}$ is a $Q$-value of $g$, so that $M=M_{i}$. We have then that every regular subgroup of $(G, Q)$ is the value of a special element which according to [6, Theorem 3.9] says that $(G, Q)$ is finite valued.

Now $a>b$ (rel. $Q$ ) so every $Q$-value of $b$ is contained in a $Q$-value of $a$; we must show this containment is proper. If not so, $a$ and $b$ have a $Q$-value in common, say $K$. By the remarks above $K$ is the $Q$-value of precisely one $a_{i}$ and one $b_{j}$; this contradicts (ii). Thus $a \gg b$ (rel. $Q$ ), and once again by (1.3.1) we conclude that $Q$ is very essential over $P$.

Let $(G, P)$ be an $l$-group; it is said to be representable if there is an $l$-isomorphism of $(G, P)$ into a cardinal product of totally ordered groups. We quote some conditions which are equivalent to the definition of representability. The proofs may be found in [8, Theorem 1.8].

(1) If $a \in P$ and $a \wedge(-x+a+x)=0$, then $a=0$.

(2) If $a \wedge b=0$ then $a \wedge(-x+b+x)=0$, for all $x \in G$.

(3) There is a family of normal prime subgroups with 0 intersection.

(4) Minimal prime subgroups are normal.

Also, if $(G, P)$ is representable then it is normal valued [8, p. 32].

(1.5) THEOREM. Let $(G, P)$ be a representable l-group; there is a very essential extension of $P$ which is totally ordered. Moreover, $P$ is the intersection of the total very essential extensions of $P$.

Proof. We may assume that $(G, P) \subseteq \Pi\left(I, K_{i}\right)$, where each $K_{i}$ is a totally ordered group, and the containment is as an $l$-subgroup. Let $I_{0}$ denote a well-ordering of the index set $I$, and let $\mathscr{S}$ denote the set of permutations of $I$. Let $I_{\xi}$ be the wellordering induced on $I$ from $I_{0}$ by applying $\xi \in \mathscr{S}$. We then order $\Pi\left(I, K_{\mathfrak{i}}\right)$ lexicographically, once for each $I_{\xi}$. Let $Q_{\xi}$ be the order induced on $G$ by the lexicographic order from $I_{\xi}$. Then $Q_{\xi}$ is a total order, and it is clear that $P$ is the meet of all the $Q_{\xi}$.

Finally each $Q_{\xi}$ is a very essential extension of $P$; for suppose $x, y \in P$ with 
$x \wedge_{P} y=0$ and $x>y$ (rel. $\left.Q_{\xi}\right)$. Let $i(x)$ (resp. $\left.i(y)\right)$ be the maximal component of $x$ (resp. $y$ ) relative to $Q_{\xi}$; since $x>y$, this implies that $i(x) \geqq i(y)$ (rel. $I_{\xi}$ ). But $x$ and $y$ are $P$-disjoint, so $i(x)$ and $i(y)$ are distinct and therefore $i(x)>i(y)$, which means that $x \gg y\left(\right.$ rel. $\left.Q_{\xi}\right)$.

Let $(G, P)$ be an $l$-group and $Q$ be an $l$-extension; we say that $P$ preserves the disjointness of $Q$ (notation: $P \mid Q$ ) if for all $x, y \in Q$ with $x \wedge_{Q} y=0$, we get that $|x|_{P} \wedge_{P}|y|_{P}=0$. (Of course, $|x|_{P}$ denotes the absolute value of $x$ relative to $P$.) We remark in passing that if $x, y \in P$ and $x \wedge_{Q} y=0$ then, without any added assumptions, $x \wedge_{P} y=0$.

(1.6) Proposition. Let $Q$ be an essential l-extension of the l-cone $P$; then $P \mid Q$.

Proof. Suppose $x$ and $y$ are $Q$-disjoint elements of $G$; since $Q$ is essential over $P$, $a \sim a_{P}^{+}$, for all $a \in Q$. This implies that $x_{P}^{+}$and $y_{P}^{+}$are $Q$-disjoint also. Since the $P$-positive parts of $x$ and $y$ exceed their respective $P$-negative parts, relative to $Q$, we conclude that both $x_{P}^{+}$and $x_{P}^{-}$are $Q$-disjoint to both $y_{P}^{+}$and $y_{P}^{-}$. By our remark preceding this proposition it follows that the above pairs are also $P$-disjoint; but then trivially $|x|_{P} \wedge_{P}|y|_{P}=0$. This completes the proof.

The following remark phrases the definition of preservation of disjointness in terms of the values of an element. The proof is trivial and will therefore be omitted.

(1.7) Lemma. Let $Q$ be an l-extension of $P$; then $P \mid Q$ if and only if we have for each $0 \neq x \in G$ that no $P$-value of $x_{Q}^{+}$is comparable to a $P$-value of $x_{Q}^{-}$.

(1.8) THEOREM. Let $Q$ be an l-extension of $P$ such that $P \mid Q$; then for each $x \in G$,

$$
x_{Q}^{+} \leqq x_{P}^{+} \text {relative to } P \text {. }
$$

Condition (*) is equivalent to

$$
a \vee_{Q} b \leqq a \vee_{P} b \text { relative to } P, \text { for all } a, b \in Q \text {. }
$$

(Note. For an l-extension $Q$ of $P$ we always have that $x_{Q}^{+} \leqq x_{P}^{+}($rel. $Q)$.)

Proof. Let $C$ be a $P$-value of $z=x_{P}^{+}-x_{Q}^{+}\left(\right.$if $x_{P}^{+} \neq x_{Q}^{+}$); there are two possibilities: $x_{P}^{+} \notin C$ or $x_{Q}^{+} \notin C$.

Case I. $x_{P}^{+} \notin C$. Then $C$ is contained in a $P$-value $K$ of $x_{P}^{+}$, and hence of $x$. Either $x_{Q}^{+}$or $x_{Q}^{-}$is in $C$; for $P \mid Q$, and so the $P$-absolute values of $x_{Q}^{+}$and $x_{Q}^{-}$are $P$-disjoint, and of course, $C$ is a $P$-prime. If $x_{Q}^{+} \in C \subseteq K$ then $x_{Q}^{-} \notin K$; so $x_{Q}^{-} \notin C$ and we have

$$
C+z=C+x-x_{Q}^{+}=C-x_{Q}^{+}+x=C+x=C+x_{P}^{+}>C .
$$

If $x_{Q}^{-} \in C$ then

$$
C+z=C+x-x_{Q}^{+}=C-x_{Q}^{-}=C,
$$

a contradiction, since $C$ is a value of $z$. 
Case II. $x_{Q}^{+} \notin C$. Then $x_{Q}^{-} \in C$, and so in view of the argument in Case I we conclude that $x_{P}^{+} \in C$; thus

$$
C+z=C-x_{Q}^{+}=C-x=C+x_{P}^{-}>C .
$$

We have therefore shown that every $P$-value of $z$ is positive (rel. $P$ ), and hence $z=x_{P}^{+}-x_{Q}^{+} \in P$.

The converse of (1.8) is false, as shown by the example (1-A): $G=\boldsymbol{R}^{2}$, $P=\{(x, y): x, y \geqq 0\}$ and $Q=\{(x, y-x): x, y \geqq 0\}$. Then $Q$ over $P$ satisfies the condition (*) in (1.8). For each element of $G$ can be put uniquely in the form $(x, y-x)$. Now let $x^{\prime}=\max \{x, 0\}$ and $y^{\prime}=\max \{y, 0\}$; then, letting $g=(x, y-x)$, we get that $g_{Q}^{+}=\left(x^{\prime}, y^{\prime}-x^{\prime}\right)$, while $g_{P}^{+}=\left(x^{\prime}, \max \{y-x, 0\}\right)$. Thus

$$
g_{P}^{+}-g_{Q}^{+}=\left(0, \max \{y-x, 0\}-\left(y^{\prime}-x^{\prime}\right)\right) \in P .
$$

However $(1,-1) \wedge_{Q}(0,1)=0$, but $|(1,-1)|_{P}=(1,1) \geqq(0,1)$ (rel. $\left.P\right)$, showing that $P \nmid Q$.

It is unknown whether condition (*) in (1.8) holds for every $l$-extension $Q$ of $P$.

(1.8.1) COROllary. If $Q$ is an essential l-extension of the l-cone $P$, then $\left(^{*}\right)$ in (1.8) holds.

(1.9) Proposition. Let $(G, P)$ be a representable l-group and $Q$ be an l-extension of $P$ such that $P \mid Q$; then $(G, Q)$ is representable.

Proof. Suppose $a \in Q$ and $a \wedge_{Q}(-x+a+x)=0$; since $P \mid Q$ we get that

$$
|a|_{P} \wedge_{P}|-x+a+x|_{P}=|a|_{P} \wedge_{P}\left(-x+|a|_{P}+x\right)=0,
$$

which implies that $|a|_{P}=0$, and hence that $a=0$, proving that $(G, Q)$ is also representable.

(1.9.1) Corollary. An essential l-extension of a representable l-cone is again a representable l-cone.

The above corollary suggests an interesting question, the answer to which turns out to be no. Suppose $(G, P)$ is an $l$-group and $Q$ is a (very) essential $l$-extension of $P$ such that $(G, Q)$ is representable. Is $(G, P)$ then also representable? Consider the following example:

(1-C) Let $F=\cdots \oplus Z_{-2} \oplus Z_{-1} \oplus Z_{0} \oplus Z_{+1} \oplus \cdots$. (Note: for each integer $n$, $Z_{n}=\boldsymbol{Z}$.) Let $G=\boldsymbol{Z} \times F$ with the following addition:

$$
\left(a, \ldots, b_{n}, \ldots\right)+\left(c, \ldots, d_{n}, \ldots\right)=\left(a+c, \ldots,\left(b_{n-c}+d_{n}\right), \ldots\right) .
$$

This makes $G$ into a group; and if we let

$$
P=\left\{\left(a, \ldots, b_{n}, \ldots\right): a>0 \text {, or } a=0 \text { and } b_{n} \geqq 0 \text {, all } n\right\}
$$

then $(G, P)$ becomes an $l$-group. $G$ is the splitting extension of $F$ by $Z$ (see [4, pp. $179-180])$. Notice that $(G, P)$ is not representable; just let $x=(1, \ldots, 0,0,0, \ldots)$ 
and $y=(0, \ldots, 0,1,0, \ldots)$ (the number 1 is in the 0 th position). It is easy to verify that $-x+y+x$ differs from $y$ only in that $-x+y+x$ has the entry 1 in the +1 position. Clearly then $y \wedge_{P}(-x+y+x)=0$.

But now let $Q=\left\{\left(a, \ldots, b_{n}, \ldots\right): a>0\right.$, or $a=0$ and the first nonzero component is positive\}. Trivially $Q \supset P$, and we check that $Q$ is a cone; once this is done it is immediate that $(G, Q)$ is a totally ordered group and $Q$ is very essential over $P$. It is quite obvious that $Q$ is a subsemigroup of $G$, so we need only establish that it is a normal one. So let $g \in G, z \in Q$; let $z=\left(a, \ldots, b_{n}, \ldots\right)$ and $g=\left(c, \ldots, d_{n}, \ldots\right)$. Then

$$
-g+z+g=\left(a, \ldots, b_{n-c}+d_{n}-d_{n-a}, \ldots\right) .
$$

So if $a>0$ there is no problem, $-g+z+g$ is clearly in $Q$. If $a=0$ then

$$
-g+z+g=\left(0, \ldots, b_{n-c}, \ldots\right),
$$

and if the first (nonzero) component of $\left(\ldots, b_{n}, \ldots\right)$ is positive, the first component of $\left(\ldots, b_{n-c}, \ldots\right)$ is also.

This example shows that the answer to the above question is indeed no. Some conditions on an $l$-group will force representability in the presence of a totally ordered very essential extension. We give one result in this connection. Recall that an $l$-group satisfies property $(\mathrm{F})$ if every positive element exceeds no more than a finite number of disjoint elements. Conrad showed (see [5]) that an $l$-group has this property if and only if every principal convex $l$-subgroup has a finite basis. In particular then, an $l$-group with property $(\mathrm{F})$ is finite valued in view of Theorem 3.7 in [6].

(1.10) Proposition. Let $(G, P)$ be an l-group satisfying property (F) and whose root system $\mathscr{R}(P)$ of $P$-regular subgroups satisfies the ascending chain condition; if $P$ has a total very essential extension then $(G, P)$ is representable.

Proof. Suppose $a \in P \mid 0$ and $x \in G$ such that $a \wedge_{P}(-x+a+x)=0$. We will show this leads to a contradiction. It clearly suffices, by our remarks above, to take a $P$-special; we also take $x$ to be $P$-special for the moment, say $x \in P$. If the $P$-values of $x$ and $a$ are incomparable then $x$ and $a$ are $P$-disjoint, and hence they commute; this is impossible in view of our assumptions. The $P$-values of $x$ and $a$ may not coincide either; for a finite valued $l$-group is normal valued, so if $x$ and $a$ have the same $P$-value, then $a$ and $-x+a+x$ also have the same $P$-value, once again a contradiction.

Now since $Q$ is very essential over $P$ we must have $a \ll-x+a+x$ or else $-x+a+x \ll a$. Let us take the first case: let $A, B$ and $D$ denote the $Q$-values of $a$, $-x+a+x$ and $x$ respectively; we are assuming that $A \subset B$. If $D \subset A$ then $x \in A$ and so $-x+a+x \in \bar{A} \backslash A$ since $a \in \bar{A} \backslash A$, a contradiction. If $A \subseteq D \subset B$ then $x \ll-x+a+x$ (rel. $Q$ ), which implies that $x \ll a$ (rel. $Q$ ), again a contradiction. Of course, if $D=B$ then since $B$ is the $Q$-value of both $x$ and $-x+a+x$, and $B$ is normal in $\bar{B}$, it follows that $B-x+a+x=B+a=B$, once more a contradiction. Thus $A \subset B \subset D$, 
or $a \ll-x+a+x \ll x$. (All inequalities in this last paragraph are to be taken relative to $Q$. Notice that the fact that $x$ is $P$-special was not used here.)

Let us denote $-x+a+x$ by $a^{x}$. With regard to the discussion preceding the last paragraph we conclude that the $P$-value of $a$ is properly contained in that of $x$. Reversing the roles of $a$ and $a^{x}$ we find that the same is true of the $P$-value of $a^{x}$. In fact, if we consider the sequence $a, a^{x}, a^{2 x}, \ldots$ we see that it has all of the following properties:

(1) $a^{n x} \wedge_{P} a^{(n+1) x}=0$, all $n=1,2, \ldots$

(2) $a^{n x} \ll x$ (rel. $P$ ), all $n$.

(3) $a \ll a^{x} \ll a^{2 x} \ll \cdots \ll x$ (rel. $Q$ ).

(4) Each $a^{n x}$ is $P$-special.

In view of property $(\mathrm{F})$ there must be a least positive integer $m$ for which $a^{m x} \wedge_{P} a^{k x}>0$, for a suitable $k<m$. Combining (3) and (4) above we obtain that $a^{k x} \ll a^{m x}$ (rel. $P$ ). In this way we build ascending chains of $P$-special elements to which correspond ascending chains of $P$-regular subgroups, one of which must be infinite since the sequence $a, a^{x}, a^{2 x}, \ldots$ consists of distinct terms. This is the contradiction we desired. If it should be the case that $a^{x} \ll a(\mathrm{rel} . Q)$ instead, simply reverse the roles of $a$ and $a^{x}$.

Suppose now that $x$ is not $P$-special; we still have that $a \ll|x|_{Q}$ (rel. $Q$ ) and that the $P$-value of $a$ is comparable to some $P$-value of $x$. Thus the $P$-value of $a$ cannot exceed all the $P$-values of $x$, and so it must be beneath exactly one of them; it is incomparable to the remaining $P$-values of $x$. Thus $a^{x}=a^{x^{\prime}}$, where $x^{\prime}$ is that $P$-special component of $x$ corresponding to the $P$-value of $x$ in which the $P$-value of $a$ is contained. This reduces the situation to the one considered previously. The proof of the proposition is therefore complete.

If the existence of a total very essential extension does not force representability on an $l$-group, then what can one say about such $l$-groups? It is tempting to say that an $l$-group $(G, P)$ which admits a total very essential extension is normal valued, in view of the following:

(1.11) Proposition. If $(G, P)$ is an l-group and $P$ is the meet of total orders then $(G, P)$ is normal valued.

Proof. We need a theorem of Wolfenstein (see [13]) which says that an l-group $(G, P)$ is normal valued if and only if $a+b \leqq 2 b+2 a$ for all $a, b \in P$. Suppose $P=\bigcap\left\{Q_{i}: i \in I\right\}$, where each $Q_{i}$ is a total order on $G$; of course $\left(G, Q_{i}\right)$ is normal valued for each $i \in I$, and so $a+b \leqq 2 b+2 a$ (rel. $Q_{i}$ ) for all $a, b \in P$. This clearly implies that $a+b \leqq 2 b+2 a$ (rel. $P$ ).

The next theorem comes close to saying that an l-group $(G, P)$ which admits a very essential extension $Q$ which is total is in fact normal valued. It is unknown whether the proof can be improved to show this.

(1.12) THEOREM. Let $(G, P)$ be an l-group, and suppose $P$ has a total very essential extension $Q$; then every nonzero element of $G$ has a normal $P$-value. 
Proof. Let $D \in \mathscr{R}(Q), 0<x \in \bar{D} \backslash D$ (rel. $P$ ). Let $C$ be the unique $P$-value of $x$ that contains $D$. Define a map $\eta: \mathscr{R}(Q) \rightarrow \mathscr{R}(P)$ by $C=D \eta$; we must show this map is well defined. So suppose $y \in \bar{D} \backslash D$ (rel. $P$ ); let $C_{x}=C$ and $C_{y}$ be the unique $P$-values of $x$ and $y$ respectively that contain $D$. We are to show that $C_{x}=C_{y}$; by way of contradiction, suppose this is not so. First, $C_{x}$ and $C_{y}$ are comparable, for if not, then, since $D$ is normal in $\bar{D}, D$ is the $Q$-value of $x+y$; moreover $y \in C_{x}$, otherwise $C_{x}$ is contained in some $P$-value of $y$ which is incomparable to $C_{y}$, thus contradicting the fact that $Q$ is very essential over $P$. Similarly $x \in C_{y}$. Therefore $C_{x}$ and $C_{y}$ are both $P$-values of $x+y$, and both contain $D$, again a contradiction.

So suppose $C_{x} \subset C_{y}$; we may assume that $x>y$ (rel. $Q$ ) by quietly taking a suitable multiple of $x$. (Keep in mind that $x$ and $y$ are Archimedean equivalent relative to $Q$.) The assumption that $C_{x} \subset C_{y}$ excludes that $x>y$ (rel. $P$ ), and of course, $x \nless y$ (rel. $P$ ). Let $a=x \wedge_{P} y$, and $x^{\prime}=x-a, y^{\prime}=y-a$; then $x^{\prime}$ and $y^{\prime}$ are nonzero elements which are $P$-disjoint. Thus either $x^{\prime} \ll y^{\prime}$ or $y^{\prime} \ll x^{\prime}$ (rel. $Q$ ); the first inequality is obviously impossible, and so we conclude that indeed $y^{\prime} \ll x^{\prime}$. Since $\bar{D} \in \mathscr{C}(P)$ we know at least that $a \in \bar{D}$.

Case I. $a \in D$; then $D+y^{\prime}=D+y$ and $D+x^{\prime}=D+x$, therefore $y^{\prime} \ll x^{\prime}$ implies that $y \ll x$, a contradiction.

Case II. $a \in \bar{D} \backslash D$; since $0 \leqq a \leqq x \in C_{y}$ (rel. $P$ ), we get that $a \in C_{y}$ and so $y^{\prime} \notin C_{y}$; in particular $y^{\prime} \notin D$. Then $D$ must be the $Q$-value of $y^{\prime}$, and by a similar argument, of $x^{\prime}$ also. In this instance then $y^{\prime} \ll x^{\prime}$ implies that $D+y^{\prime} \ll D+x^{\prime}$, once more a contradiction.

Conclusion. $C_{x} \notin C_{y}$, and by symmetry $C_{y} \notin C_{x}$; hence $C_{x}=C_{y}$. We established that the map $\eta$ defined in the first paragraph of the proof is well defined. We claim that $\eta$ is $1-1$ and its inverse preserves order.

So suppose $D, D^{\prime} \in \mathscr{R}(Q)$ and $D \eta \subseteq D^{\prime} \eta$; we will show that $D \subseteq D^{\prime}$. By way of contradiction suppose $D^{\prime} \subset D$. (Note: $(G, Q)$ is totally ordered.) We prove first that $D=D \eta \cap \bar{D}$, for all $D \in \mathscr{R}(Q)$; this is more or less clear, since by definition $x$ $\in \overline{D \eta} \backslash D \eta$ if $x \in \bar{D} \backslash D$, and of course $D \subseteq D \eta \cap \bar{D}$. Now $\bar{D}^{\prime} \subseteq D$, and hence $\bar{D}^{\prime} \subseteq D \eta$ $\subseteq D^{\prime} \eta$, which implies that $D^{\prime}=D^{\prime} \eta \cap \bar{D}^{\prime}=\bar{D}^{\prime}$, a contradiction. Thus $D \subseteq D^{\prime}$; in particular $D \eta=D^{\prime} \eta$ implies that $D=D^{\prime}$, showing that $\eta$ is $1-1$.

Next let $C=D \eta ;\langle\bar{D}, C\rangle$ is in $\mathscr{C}(P)$, and obviously $\bar{D} \subseteq \bar{C}$. Therefore $\langle\bar{D}, C\rangle$ is a convex $l$-subgroup of $\bar{C}$ that contains $C$ properly, and so $\bar{C}=\langle\bar{D}, C\rangle$. We now show that $\bar{D}$ and $C$ are permutable; moreover, we prove that for all $d \in \bar{D}$ and $c \in C, d+c-d \in C$. Once this is done it becomes clear that $\bar{D}+C=C+\bar{D}$ and hence that $C$ is normal in $\langle\bar{D}, C\rangle=\bar{D}+C=\bar{C}$. We also get for free that

$$
\bar{C} / C=\bar{D}+C / C \cong \bar{D} / \bar{D} \cap C=\bar{D} / D \text { (with order). }
$$

In any case the theorem will have been proved.

We now return to the proof of our claim. We have the following identity (see [9]):

$$
d-d \wedge_{P} c+c=d \vee_{P} c
$$


for all $d, c \in G$. If $d \in \bar{D}$ and $c \in C$, then $d \wedge_{P} c \in D$, which implies that $d-d \wedge_{P} c$ $=-k+d$, for some $k \in D$. (Remember: $D$ is normal in $\bar{D}$.) In any case

$$
-k+d+c=d \vee_{P} c=c \vee_{P} d=c-c \wedge_{P} d+d,
$$

and so $d+c=-k+c-c \wedge_{P} d+d$, and $-k+c-c \wedge_{P} d \in C$. This shows what we wanted.

REMARK. The theorem shows that every nonzero element of $G$ has a normal $P$-value. In such a case a nonzero element $x$ is $P$-positive if and only if all of its normal values are $P$-positive.

If one could show in the proof of this theorem that $\eta$ is onto $\mathscr{R}(P)$, then of course every $P$-regular subgroup would be normal in its cover; hence $(G, P)$ would be normal valued. Wolfenstein proves in [13] that the following conditions are equivalent to normal valuedness:

(1) There is a plenary subset of $\mathscr{R}(P)$, say $\mathscr{R}$, consisting of normal values. ( $\mathscr{R}$ is a plenary subset of $\mathscr{R}(P)$ if $\bigcap \mathscr{R}=0$, and $\mathscr{R}$ is a dual ideal of $\mathscr{R}(P)$.)

(2) For all $a, b \in P, a+b \leqq 2 b+2 a$.

(3) $A+B=B+A$, for all convex $l$-subgroups $A$ and $B$ of $(G, P)$.

The author was unable to extract any one of these conditions from the proof of (1.12).

Recall the definitions of the three "radicals" used in the theory of $l$-groups (see [1]). Let $(G, P)$ be an $l$-group; for each $g \neq 0$, let $R_{g}$ (resp. $\left.L_{g}\right)$ be the join of all convex $l$-subgroups (resp. $l$-ideals) of $(G, P)$ that do not contain $g$. Then define

and

$$
R(G, P)=\bigcap\left\{R_{g}: g \neq 0\right\}
$$

$$
L(G, P)=\bigcap\left\{L_{g}: g \neq 0\right\} .
$$

Since $L_{g} \subseteq R_{g}$, for all $g \in G \backslash 0$, we see that $L(G, P) \subseteq R(G, P)$. Byrd and Lloyd defined the so-called distributive radical $D(G, P)$ as the intersection of all closed prime subgroups. They showed, among other things, that

(1) $L(G, P) \subseteq D(G, P) \subseteq R(G, P)$ in general;

(2) $(G, P)$ is a completely distributive $l$-group if and only if $D(G, P)=0$;

(3) if $(G, P)$ is representable then all the radicals coincide;

(4) if $(G, P)$ is normal valued then $D(G, P)=R(G, P)$.

Suppose now that $Q$ is an essential $l$-extension of $P$; then $\mathscr{C}(Q) \subseteq \mathscr{C}(P)$, and so it follows immediately that $L(G, P) \supseteq L(G, Q)$ and $R(G, P) \supseteq R(G, Q)$. Also $D(G, P)$ $\supseteq D(G, Q)$, for suppose $K$ is a $Q$-closed, $Q$-prime subgroup; $K \in \mathscr{C}(P)$ and is therefore contained in a $P$-prime, which in turn is contained in a $P$-closed, $P$-prime subgroup (which could be $G$ itself). Using (2) above we can conclude:

(1.13) Proposition. If $(G, P)$ is a completely distributive l-group and $Q$ is an essential l-extension of $P$, then $(G, Q)$ is also completely distributive.

Finally we give an example of an $l$-group whose cone has no proper very essential $l$-extensions. 
(1-D) Let $G=\boldsymbol{Z} \times \boldsymbol{Z} \times \boldsymbol{Z}$ with the following addition:

$$
\begin{aligned}
\left(a_{1}, a_{2}, a_{3}\right)+\left(b_{1}, b_{2}, b_{3}\right) & =\left(a_{1}+b_{1}, a_{2}+b_{2}, a_{3}+b_{3}\right), \\
& =\left(a_{2}+b_{1}, a_{1}+b_{2}, a_{3}+b_{3}\right),
\end{aligned}
$$

according to whether $b_{3}$ is even or odd. Let $P=\left\{\left(a_{1}, a_{2}, a_{3}\right): a_{3}>0\right.$, or else $a_{3}=0$ and $\left.a_{1}, a_{2} \geqq 0\right\}$. Then it is known that $(G, P)$ is an $l$-group, and one can show that $G$ admits no total orders (see [8]).

Suppose $Q \supset P$ is a very essential $l$-extension of $P$; then there exist $a, b \in P$ such that $a \wedge_{P} b=0$ and $a \gg b$. Since $a$ and $b$ are $P$-disjoint we may assume that $a$ $=\left(a_{1}, 0,0\right)$ and $b=\left(0, b_{1}, 0\right)$; and by taking a suitable multiple, we may suppose that $b_{1}>a_{1}$ in $Z$, while still $a \gg b$ (rel. $Q$ ). Let $z=(0,0,1)$; then $-z+b+z=\left(b_{1}, 0,0\right)$ $>a($ rel. $P)$. Yet on the other hand $-z+b+z \ll-z+a+z<b \ll a$ (rel. $Q)$; this is a contradiction, and we can conclude that $P$ admits no proper very essential $l$ extensions.

2. Essential extensions of partially ordered abelian groups. In this section all groups will be abelian; all partial orders will be semiclosed; that is, a positive multiple of the element $g$ is positive in the group only if $g$ itself is positive. We will also require that all partially ordered groups be Riesz groups; a partially ordered group (henceforth p.o. group) is said to be Riesz if it satisfies the Riesz interpolation property: for $a, b, c, d \in G$ with $a, b \leqq c, d$, there exists an $x \in G$ such that $a, b \leqq x$ $\leqq c, d$. We also require that all groups be directed.

A p.o. group $(G, P)$ is a pseudo lattice group (abbr. pl-group) if every nonzero element $g$ can be written as $g=a-b$, where $a$ and $b$ are in $P$, and no value of $a$ is comparable to a value of $b$. Two such elements are called pseudo disjoint (abbr. $p$-disjoint). It is immediate that a $p l$-group is directed, and Teller [12] has shown it is in fact Riesz. For the fundamental results on pl-groups and Riesz groups see [7] and [10] respectively. Some references will also be made to [3].

By a Riesz-extension $Q$ of the Riesz-cone $P$ we will mean an extension which is again Riesz; we shall abbreviate to Rz-cone and Rz-extension, etc. A Rz-extension $Q$ of $P$ is said to be essential over $P$ if every o-ideal of $(G, Q)$ is an o-ideal of $(G, P)$. We quote without proofs the following analogues of results in $\S 1$.

(1) The relation "essential over" is transitive; moreover, if $Q^{\prime}$ is essential over $P$, and $Q^{\prime} \supseteq Q \supseteq P$, then $Q^{\prime}$ is essential over $Q$.

(2) Suppose $(G, P)$ is a $p l$-group; then $Q$ is an essential Rz-extension of $P$ if and only if, for each $x \in Q$ and $x=a-b$, a representation of $x$ with $a$ and $b p$-disjoint in $(G, P)$, we get that $x \sim a$ (rel. $Q)$.

(3) (Structure Lemma). If $Q$ is an essential Rz-extension of $P$, then for each nonzero element $x$ and each $Q$-value $D$ of $x$, there is a $P$-value $C$ of $x$ which contains $D$ and no other $Q$-value of $x$.

With regard to (3) we might point out that in order to imitate the proof given in $\S 1$, we find it very useful that for Riesz groups it turns out that the sum of two 
o-ideals is again an o-ideal. The following proposition also indicates that the assumption that everything in this discussion is at least Riesz is a pleasant one.

(2.1) Proposition. Let $(G, P)$ be a pl-group and $\left\{Q_{i}: i \in I\right\}$ be a chain of essential Rz-extensions of $P ; Q=\bigcup\left\{Q_{i}: i \in I\right\}$ is an essential Rz-extension of $P$. Thus a maximal Rz-extension exists.

Proof. First of all $Q$ is a Riesz-cone, for if $a, b, c, d \in G$ with $a, b \leqq c, d$ (rel. $Q$ ) then since the $Q_{i}$ form a chain, this inequality holds relative to some $Q_{j}(j \in I)$. But each $Q_{i}$ is Riesz, so that we get an $x \in G$ such that $a, b \leqq x \leqq c, d$ (rel. $Q_{j}$ ), and hence also relative to $Q$.

Now suppose $g \in Q$ and $g=a-b$, with $a$ and $b p$-disjoint in $(G, P)$. Then $g \in Q_{k}$ for some $k \in I$, and so because $Q_{k}$ is essential over $P$, we conclude that $g \sim a\left(\right.$ rel. $Q_{k}$ ), and hence relative to $Q$.

We note that maximal essential Rz-extensions need not be totally ordered. Consider the following small example:

(2-A) Let $(G, P)=Z \boxplus Z$, and $Q=\{(m, n): m+n>0\} . Q$ is an essential extension of $P$ because $(G, Q)$ is o-simple. Suppose, by way of contradiction, that $Q^{\prime} \supset Q$ is an essential Rz-extension of $P$. Clearly every element in $Q$ exceeds, relative to $Q$, any $Q^{\prime}$-positive element on the diagonal $D=\{(m, n): m=-n\}$. Therefore this diagonal is a convex subgroup of $\left(G, Q^{\prime}\right)$. Now $Q^{\prime} \neq Q$ so $(m,-m)$ $\in Q^{\prime}$, for some nonzero integer $m$; since $Q^{\prime}$ is semiclosed we get that $(1,-1) \in Q^{\prime}$ or else $(-1,1) \in Q^{\prime}$. In any case $D$ is a totally ordered subgroup, and hence an o-ideal. This implies that $D$ is an $l$-ideal of $(G, P)$ which is of course absurd. (Note: $Q^{\prime}$ is in fact $\{(m, n): m+n>0$, or $m=-n$ and $m \geqq 0\}$ or $\{(m, n): m+n>0$, or $m=-n$ and $n \geqq 0\}$.)

Let $(G, P)$ be a $p l$-group and $Q$ be an essential Rz-extension of $P$; consider a regular o-ideal of $(G, Q)$, say $D$. Then $D$ is maximal without some $g \neq 0$; write $g=a-b$, with $a$ and $b p$-disjoint in $(G, P)$. Pick a $P$-value $C$ of $g$, containing $D$; we may assume that $C+g=C+a>C$ (rel. $P$ ). Thus $a \notin D$, and $D$ is in fact a $Q$-value of $a$; for if $M$ is an o-ideal of $(G, Q)$ properly containing $D$, then $g \in M$, and since $M$ is also an o-ideal of $(G, P)$ it follows that $a \in M$. Therefore $D+a>D$ (rel. $Q$ ), and so by a result of Conrad [7, §3] the intersection $\bar{D}$ of all the o-ideals of $(G, Q)$ that properly contain $D$ is again an o-ideal of $(G, Q)$. The quotient $\bar{D} / D$ is o-simple; if $\bar{D} / D$ is divisible (in particular if $G$ is) then it is anti-lattice (i.e. a Riesz group in which the only joins and meets that exist are the trivial ones).

The following lemma is the analogue for $p l$-groups of the decomposition of elements into their special components in finite valued $l$-groups.

(2.2) Lemma. Let $(G, P)$ be a finite valued pl-group; let $0<g \in G$ and $C_{1}, \ldots, C_{n}$ be its values. Then there exists a special element $h$ such that $C_{1}+g=C_{1}+h$, and the values of $g-h$ are precisely $C_{2}, \ldots, C_{n}$.

Proof. (Induction on the number of values.) Choose $k<0$ as follows: $C_{2}$ is its only value [7, Proposition 4.10], and $C_{2}+g+k<C_{2}$; the latter is possible since 
$\bar{C}_{2} / C_{2}$ is an Archimedean o-group. The values of $g+k$ are precisely $C_{1}, C_{2}, \ldots, C_{n}$, and $C_{j}+g+k=C_{j}+g$, for $j \neq 2$. Write $g+k=a-b$, with $a$ and $b p$-disjoint; then $C_{2}$ is the only value of $b$, and $C_{2}+g+k=C_{2}-b$; the values of $a$ are the remaining $C_{j}$, and $C_{j}+g+k=C_{j}+a(j \neq 2)$. It follows that $C_{2}+b+k=C_{2}-g$, and so $b+k$ is special at $C_{2}$.

By induction we can find a special element $h \in G$ such that $C_{1}+h=C_{1}+a=C_{1}+g$, and the values of $a-h$ are exactly $C_{3}, \ldots, C_{n}$. Consider now $g-h=(a-h)-(b+k)$; $b$ and $k$ are both in $C_{j}(j \geqq 3)$, so all these $C_{j}$ 's are values of $g-h$. On the other hand $a-h \in C_{2}$, so that $C_{2}$ is also a value of $g-h$. It is a simple matter to check that these are the only values of $g-h$.

This completes the proof of the lemma.

(2.2.1) Corollary. Let $(G, P)$ be a finite valued pl-group; let $C_{1}, C_{2}, \ldots, C_{n}$ be the values of $0<g \in G$. Then there exist positive special elements $g_{1}, g_{2}, \ldots, g_{n}$ such that for each $j=1,2, \ldots, n, C_{j}$ is the value of $g_{j} ; g_{i}$ and $g_{k}$ are $p$-disjoint if $i \neq k$; and finally $g=g_{1}+\cdots+g_{n}$.

REMARK. In the context of Corollary (2.2.1) there is also a decomposition into special elements for an arbitrary nonzero element $g$. We write $g=a-b$, with $a$ and $b$-disjoint, and use the decompositions of $a$ and $b$ to get $g=g_{1}+\cdots+g_{n}$, where each $g_{j}$ is special, and $\left|g_{i}\right|$ and $\left|g_{j}\right|$ are $p$-disjoint if $i \neq j$ (by $\left|g_{j}\right|$ we mean $g_{j}$ or $-g_{j}$, whichever is positive).

(2.3) Proposition. Let $(G, P)$ be a finite valued pl-group, and $Q$ be an essential Rz-extension; let $g \neq 0$ and $D$ be a $Q$-value of $g$. If $C+g>C$ (rel. $P$ ) for each $P$-value of $g$ containing $D$, then $D+g>D($ rel. $Q)$.

Proof. Let $g=g_{1}+\cdots+g_{n}$ be a decomposition of $g$ into $P$-special components. Let $\mathscr{C}_{D}$ be the set of all $P$-values of $g$ containing $D$; note that if $C$ is a $P$-value of $g$, then $C+g>C$ if and only if the $P$-special component, which $C$ is the $P$-value of, is itself positive. Therefore if $C+g>C$, for each $C \in \mathscr{C}_{D}$, then $D+g_{i}>D$ (rel. $Q$ ) for all $g_{i}$ 's which are $P$-special at some $C \in \mathscr{C}_{D}$. If $g_{k}$ is not special at any $C \in \mathscr{C}_{D}$ then $g_{k} \in D$; for otherwise there must be a $Q$-value $D^{\prime}$ of $g_{k}$ containing $D$. Since $Q$ is essential over $P, D^{\prime}$ is contained in a $P$-value of $g_{k}$ which is then a $P$-value of $g$, and this is a contradiction. Thus $D+g=D+g_{i_{1}}+\cdots+g_{i_{t}}>D$, where the $g_{i}$ 's appearing in this sum are precisely those which are special at some $C \in \mathscr{C}_{D}$.

Let $Q$ be an essential Rz-extension of the Rz-cone $P$; $Q$ is very essential over $P$ if for each nonzero element $x$ and each $Q$-value $D$ of $x$, there is precisely one $P$-value of $x$ that contains $D$.

(2.4) THEOREM. Let $(G, P)$ be a pl-group, and $Q$ be a Rz-extension of $P$ satisfying:

(i) $g \in Q$ if and only if every $Q$-value of $g$ is $Q$-positive;

(ii) for every regular o-ideal of $(G, Q) D$, the cover $\bar{D}$ is an o-ideal and $\bar{D} / D$ is totally ordered in the induced order. 
Then $Q$ is very essential over $P$ if and only if for each $x \in Q$ and each representation $x=a-b$, with $a$ and $b$-disjoint in $(G, P)$, we have $a \gg b$ (rel. $Q)$.

Proof. (Necessity) Entirely analogous to the corresponding part in (1.3).

(Sufficiency) Let $g \in Q \mid 0$ and $g=a-b$ be a representation of $g$ as a difference of $p$-disjoint elements in $(G, P)$; let $D$ be a $Q$-value of $g$, and suppose $C_{1} \| C_{2}$ are $P$-values of $g$ that contain $D$. (At least one such $P$-value exists, since the condition obviously implies that $Q$ is essential over $P$.) Then just as in the proof of (1.3), one shows that $C_{i}+g=C_{i}+a>C_{i}($ rel. $P), i=1,2$. Also, one exhibits the existence of elements $x_{1}$ and $x_{2}$ which are $P$-positive, and such that $x_{i} \in \bar{C}_{i} \mid C_{i}, i=1,2$, and $x_{1} \in C_{2}, x_{2} \in C_{1}$. The proof of this fact is more delicate in the present situation, and so we carry it out in detail.

(All inequalities in the remainder of this proof are to be taken relative to $P$.)

Since $C_{1} \| C_{2}$ we can pick $0<c \in C_{2} \mid C_{1}$ and $0<d \in C_{1} \mid C_{2}$ (rel. $P$ ). If $c \in \bar{C}_{1}$ we take $x_{1}=c$; so suppose this is not the case. Clearly $a$ and $c$ are not $p$-disjoint and hence not disjoint; using the Riesz interpolation property we obtain a $P$-positive element $\leqq a$ and $c$. Suppose now that whenever $0<z \leqq a, c$ (rel. $P$ ) we get that $z \in C_{1}$; then $C_{1}+a \wedge_{P} C_{1}+c=C_{1}$. For if $C_{1}+h \leqq C_{1}+a, C_{1}+c$, then there exists an $h^{\prime} \in C_{1}$ such that $h-h^{\prime} \leqq a, c$; using the interpolation property once more, we get an $h^{\prime \prime}$ such that $h-h^{\prime}, 0 \leqq h^{\prime \prime} \leqq a, c$. By our assumption $h^{\prime \prime} \in C_{1}$, so since $h \leqq h^{\prime}+h^{\prime \prime} \in C_{1}$, we conclude that $C_{1}+h \leqq C_{1}$.

But Conrad has shown [7, Proposition 4.6] for pl-groups that if $K$ is a regular o-ideal, $K+f>K$ and $f \notin \bar{K}$, then $K+f>\bar{K} / K$. In our case it implies that $C_{1}+c$ $>C_{1}+a$, a contradiction. We must conclude therefore that there exists a positive $x_{1} \leqq a, c$ such that $x_{1} \notin C_{1}$; this is the $x_{1}$ we wanted. The element $x_{2}$ is chosen in an entirely similar manner.

From here the proof proceeds exactly as for (1.3).

(2.4.1) Corollary. Let $(G, P)$ be a finite valued pl-group, and $Q$ be a pl-extension (that is, a Rz-extension such that $(G, Q)$ is again a pl-group), then $Q$ is very essential over $P$ if and only if

(i) every $P$-special element is $Q$-special, and

(ii) whenever $x$ and $y$ are $p$-disjoint special elements in $(G, P)$ which are not $p$ disjoint in $(G, Q)$, then either $x \ll y$ or $y \ll x($ rel. $Q)$.

Proof. Imitate the proof of (1.4).

(2.5) Proposition. Let $(G, P)$ be a divisible pl-group; let $\mathscr{R}=\mathscr{R}(P)$ denote the set of regular o-ideals of $(G, P)$. We can embed $(G, P)$ in $V\left(\mathscr{R}, R_{C}\right)$ (where for each $\left.C \in \mathscr{R}, R_{C}=\bar{C} / C\right)$ in such a way that $(G, P) \pi$ is a pl-subgroup $\left({ }^{3}\right)$ of $V\left(\mathscr{R}, R_{C}\right)$, and $C$ is a value of $g \neq 0$ if and only if $g \pi_{C}$ is a maximal component of $g \pi$, and then $g \pi_{C}$ $=C+g$; of course $\pi$ is the embedding in question.

$\left({ }^{3}\right)$ A subgroup $K$ of a $p l$-group $\left(H, P^{\prime}\right)$ is a $p l$-subgroup if every $x \in K$ has a representation $x=a-b$, with $a$ and $b p$-disjoint in $\left(H, P^{\prime}\right)$ and belonging to $K$. 
Now suppose $\mathscr{R}^{\prime}$ denotes an extension of the inclusion order on $\mathscr{R}$. Suppose also that $G \pi \subseteq V\left(\mathscr{R}^{\prime}, R_{C}\right)$ as a pl-subgroup; then $Q=\left(G \pi \cap V\left(\mathscr{R}^{\prime}, R_{C}\right)^{+}\right) \pi^{-1}$ is a very essential pl-extension of $P$.

If $(G, P)$ is finite valued we need not assume that $G \pi$ is a pl-subgroup of $V\left(\mathscr{R}^{\prime}, R_{C}\right)$.

Proof. The assertion in the first paragraph is essentially the content of Theorem 4.10 in [7]. We shall pretend, without loss of generality, that $G$ is in fact contained in $V\left(\mathscr{R}, R_{C}\right)$ as explained in that paragraph.

$(G, Q)$ is a $p l$-group in its own right and so Theorem (2.4) applies. Let $x \in Q$ and write $x$ as the difference of positive $p$-disjoint elements of $(G, P)$, say $x=a-b$. Considering them as elements of $V\left(\mathscr{R}, R_{C}\right)$ we have that no maximal component of $a$ is comparable to a maximal component of $b$. But $a>b$ (rel. $Q$ ), so every maximal component of $b$ is beneath a maximal component of $a$, all relative to $\mathscr{R}^{\prime}$. But this implies that $a \gg b$ (rel. $Q$ ) because $a$ and $b$ have no $\mathscr{R}$-maximal components, and hence no $\mathscr{R}^{\prime}$-maximal components in common. This proves that $Q$ is very essential over $P$.

Assume now that $(G, P)$ is finite valued, and $(G, Q) \subseteq V\left(\mathscr{R}^{\prime}, R_{C}\right)$; we must show that $(G, Q)$ is a $p l$-subgroup of $V\left(\mathscr{R}^{\prime}, R_{C}\right)$. So let $g \in G \mid 0$ and $g=a-b$ be a representation of $g$ as a difference of $p$-disjoint elements of $(G, P)$; let $a=a_{1}+\cdots+a_{m}$ be a decomposition of $a$ into its $P$-special components, and let $a^{\prime}=a-a_{1}$. We proceed by induction on the number of values of $g($ rel. $P$ ). We can therefore write $a^{\prime}-b=c-d$, where $c$ and $d$ are $p$-disjoint in $V\left(\mathscr{R}^{\prime}, R_{C}\right)$ and also elements of $G$. Certainly, $a_{1}$ is special as an element of $V\left(\mathscr{R}^{\prime}, R_{C}\right)$; we consider $a_{1}+c$. There are three cases:

I. $a_{1}$ is $p$-disjoint to $d$ (rel. $\mathscr{R}^{\prime}$ ); then $a_{1}+c$ is $p$-disjoint to $d$ (rel. $\left.\mathscr{R}^{\prime}\right)$. (Of course $\left.g=\left(a_{1}+c\right)-d.\right)$

II. The $\mathscr{R}^{\prime}$-maximal component of $a_{1}$ lies beneath some $\mathscr{R}^{\prime}$-maximal component of $d$; in this case $c$ and $d-a_{1}$ are $p$-disjoint (rel. $\left.\mathscr{R}^{\prime}\right)$, and also $g=c-\left(d-a_{1}\right)$.

III. The $\mathscr{R}^{\prime}$-maximal component of $a_{1}$ exceeds some $\mathscr{R}^{\prime}$-maximal component of $d$; let $d=d_{1}+\cdots+d_{n}$ be a decomposition of $d$ into $\mathscr{R}^{\prime}$-special components. Let $u$ be the element of $G$ obtained by subtracting from $a_{1}$ those $d_{i}$ whose $\mathscr{R}^{\prime}$-maximal components are exceeded by that of $a_{1}$; let $v$ be the sum of the remaining $d_{i}$. Then $c+u$ is $p$-disjoint from $v$ (rel. $\mathscr{R}^{\prime}$ ), and $g=c+u-v$.

This completes the proof of the proposition.

(2.5.1) Corollary. Let $(G, P)$ be a finite valued, divisible pl-group. If $P$ is not totally ordered then a proper very essential pl-extension $Q$ of $P$ exists.

Proof. We may take $(G, P)$ to be a $p l$-subgroup of $V\left(\mathscr{R}(P), R_{C}\right)$ as in (2.5). Suppose $P$ is not totally ordered, and pick $C_{1} \| C_{2}$ in $\mathscr{R}(P)$; define a new order $\tilde{R}$ on $\mathscr{R}(P)$ by $C \leqq C^{\prime}$ if $C \subseteq C^{\prime}$, or else $C \subseteq C_{1}$ and $C_{2} \subseteq C^{\prime} . \tilde{R}$ is then a partial order extending the inclusion on $\mathscr{R}(P)$, and therefore $V\left(\mathscr{R}, R_{C}\right) \subseteq V\left(\mathscr{R}(P), R_{C}\right)$; it is easy to see that the reverse inequality also holds; just observe that an infinite ascending sequence relative to $\tilde{\mathscr{R}}$ yields at least one infinite ascending sequence relative to 
$\mathscr{R}(P)$. Then according to Proposition (2.5) $Q=G \cap V\left(\tilde{R}, R_{C}\right)^{+}$is a very essential $p l$-extension of $P$, and it is obvious that $Q$ contains $P$ properly.

The next result says that condition (ii) in the hypothesis of (2.4) is as good as we can expect, and that (i) is not so bad either. In the finite valued case it tells us that very essential extensions of $p l$-groups are "almost" $p l$-groups.

(2.6) THEOREM. Let $(G, P)$ be a pl-group and $Q$ be a very essential Rz-extension of $P$. Then

(a) Condition (ii) in (2.4) holds.

(b) There is an extension $Q^{\prime}$ of $Q$ such that $(G, Q)$ and $\left(G, Q^{\prime}\right)$ have the same $o$-ideals, and an element $g$ in $G$ is $Q^{\prime}$-positive if and only if all its $Q^{\prime}$-values are positive. Thus if $\left(G, Q^{\prime}\right)$ is Riesz then $Q^{\prime}$ is a very essential extension of $P$ which does satisfy (i) and (ii) in (2.4).

(c) If $(G, P)$ is finite valued then $\left(G, Q^{\prime}\right)$ is in fact a pl-group.

Proof. (a) Let $D \in \mathscr{R}(Q)$; we already know that the cover $\bar{D}$ of $D$ is an o-ideal of $(G, Q)$ and that $\bar{D} / D$ is o-simple. So it suffices to show that the quotient is totally ordered. Let $g \in \bar{D} \backslash D$, and $a$ and $b$ be $p$-disjoint elements in $(G, P)$ such that $g=a-b$. Then $a, b \in \bar{D}$; we claim that $D$ cannot be a $Q$-value of both $a$ and $b$. For if this is the case, then there are $P$-values $C_{a}$ and $C_{b}$ of $a$ and $b$ respectively, both containing $D$. However, $C_{a}$ and $C_{b}$ are then $P$-values of $g$, thus contradicting the fact that $Q$ is very essential over $P$. Thus $D+g=D+a$ or $D-b$; this proves that $\bar{D} / D$ is an o-group relative to $Q$, which is what we wanted.

(b) Define $x \in Q^{\prime}$ if $D+x>D$ (rel. $Q$ ) for every $Q$-value $D$ of $x$. To see $Q^{\prime}$ is a cone it clearly suffices to show it is a semigroup. Let $x, y \in Q^{\prime}$; then $x+y \neq 0$, so let $D$ be a $Q$-value of $x+y$. Without loss of generality we may assume $x \notin D$; let $M$ be a $Q$-value of $x$ containing $D$. Then $M=D$, otherwise $x+y \in M$ and therefore $y \notin M$; but since $x \in \bar{M}, y \in \bar{M}$ also, and so $M+x+y=M+x+M+y>M$, a contradiction. Thus $M=D$, and $y \in \bar{D}$, so $D+x+y>D+y \geqq D$, and so $x+y \in Q^{\prime}$.

Clearly $Q^{\prime}$ extends $Q$; we now show $(G, Q)$ and $\left(G, Q^{\prime}\right)$ have the same o-ideals. Once we have done this it becomes obvious that if $\left(G, Q^{\prime}\right)$ is Riesz, then $Q^{\prime}$ is a very essential extension of $P$ which satisfies (i) and (ii) in (2.4).

Let $K$ be an o-ideal of $\left(G, Q^{\prime}\right)$; let $a$ and $b$ be $p$-disjoint elements of $(G, P)$ and $z=a-b \in K \cap Q^{\prime}$. If $D$ is any $Q$-value of $z$ we must have $b \in D$, by the proof of (a), since every $Q$-value of $z$ is $Q$-positive. Thus $D+2 z>D+a$ (rel. $Q$ ), and the set of $Q$-values of $z$ coincides with the set of $Q$-values of $2 z-a$; consequently $2 z>a$ (rel. $Q^{\prime}$ ), and in particular $a \in K$, so $b \in K$ also. This proves $K$ is $Q$-directed, and since it is clearly $Q$-convex, it follows that $K$ is an o-ideal of $(G, Q)$.

Now let $M$ be an o-ideal of $(G, Q)$, and suppose $0<y<x \in M$ (rel. $\left.Q^{\prime}\right)$. If $y \notin M$, let $N$ be a $Q$-value of $y$ containing $M$; now $x \in N$, so $N$ is a $Q$-value of $x-y$ and $N<N+x-y=N-y<N$, which amounts to a contradiction. Therefore $y \in M$ and $M$ is $Q^{\prime}$-convex. It is already $Q^{\prime}$-directed, and thus an o-ideal of $\left(G, Q^{\prime}\right)$. 
Finally we remark that $g \in Q^{\prime}$ if and only if all the $Q^{\prime}$-values of $g$ are positive. Notice also that $Q^{\prime}$ is a semiclosed partial order.

(c) Suppose now that $(G, P)$ is finite valued. Let $g \in G \mid 0$ and $g=g_{1}+\cdots+g_{n}$ be a decomposition of $g$ into $P$-special components. Let $D_{1}, \ldots, D_{m}(m \leqq n)$ be the $Q$-values of $g$; if $g_{i} \notin D_{j}$ then $D_{j}$ must be the $Q$-value of $g_{i} ; 1 \leqq i \leqq n, 1 \leqq j \leqq m$. This is so because $g \in \bar{D}_{j}$, and therefore each $g_{i} \in \bar{D}_{j}$. Given $D_{j}$, let $g_{i(j)}$ be the unique component of $g$ which is not in $D_{j}$ (the uniqueness is a consequence of the fact that $Q$ is very essential over $P$ ). Let $g_{j}^{\prime}$ be the sum of $g_{i(j)}$ plus every $g_{k}$ whose $Q$-value lies beneath $D_{j}$ but not $D_{j-1}$. Then $g=g_{1}^{\prime}+\cdots+g_{m}^{\prime}$, and for each $j=1, \ldots, m$, $D_{j}+g=D_{j}+g_{i(j)}=D_{j}+g_{j}^{\prime}$ and $g_{j}^{\prime}$ is $Q$-special at $D_{j}$. Let $a$ be the sum of all the $g_{j}^{\prime}$ such that $D_{j}+g>D_{j}$ (rel. $Q$ ), and $b=a-g$. Then $a, b \in Q^{\prime}$ and they are obviously $p$-disjoint relative to $Q^{\prime}$. This proves that $\left(G, Q^{\prime}\right)$ is a pl-group, and the proof is complete.

Let us give a simple example to illustrate what Theorem (2.6) says:

(2-B) Let $(G, P)=\boldsymbol{R} \boxplus \boldsymbol{R}$ and $Q=\{(x, y): x+y>0$ and $x \geqq 0\} ;(G, Q)$ is a Riesz group, and $G, 0 \times \boldsymbol{R}$ and 0 are the only o-ideals of $(G, Q) . Q$ is clearly an essential extension of $P$. 0 is the $Q$-value of all $(0, y)$, and $0 \times \boldsymbol{R}$ is the $Q$-value of all remaining $(x, y)$. But relative to $P, \boldsymbol{R} \times 0$ is the unique value of all $(0, y)$, and $0 \times \boldsymbol{R}$ is the only $P$-value of $(x, y)$ (with $x \neq 0)$ that contains $0 \times \boldsymbol{R}$. Therefore $Q$ is very essential over $P$.

The element $(1,-1) \notin Q$, yet $0 \times \boldsymbol{R}+(1,-1)>0 \times \boldsymbol{R}$ (rel. $Q)$. Since every element of $G$ is $Q$-special this shows $(G, Q)$ is not a pl-group. In fact $\{(x, y): x>0$, or $x=0$ and $y \geqq 0\}$ is precisely the set of elements with positive $Q$-value (except for 0 ). According to (2.6) this set is exactly the $Q^{\prime}$ described there.

(2.7) Theorem. Let $(G, P)$ be a finite valued pl-group; let $\left\{Q_{i}: i \in I\right\}$ be a cha in of very essential pl-extensions of $P$. Then $Q=\left\{Q_{i}: i \in I\right\}$ is also very essential over $P$. Moreover, maximal very essential pl-extensions exist.

Proof. By (2.1) $Q$ is essential over $P$ and Riesz; it suffices therefore to show that if $x$ and $y$ are $p$-disjoint and special in $(G, P)$ then they do not have the same $Q$-value. If there is an $i \in I$ relative to which $x$ and $y$ are no longer $p$-disjoint in $\left(G, Q_{i}\right)$, then by (2.4.1) we may take $x \ll y$ (rel. $Q_{i}$ ). This means that $x \ll y$ (rel. $Q$ ) and so $x$ and $y$ cannot have the same $Q$-value. So we assume that $x$ and $y$ are $p$-disjoint in each $\left(G, Q_{i}\right)$, and suppose, by way of contradiction, that they do have the same $Q$-value $D$. By the o-simplicity of $\bar{D} / D$ and since $x, y \in P$, we have for a suitable positive integer $n$ that $D+y<D+n x($ rel. $Q)$; choose $d \in D$ such that $n x-y>d$. There is also a $j \in I$ such that $n x-y>d$ (rel. $Q_{j}$ ). Let $C_{x}$ and $C_{y}$ be the $Q_{j}$-values of $x$ and $y$ respectively; they must automatically contain $D$. Therefore, since $d \in D \subseteq C_{y}$ we get that $C_{y}+n x \geqq C_{y}+y>C_{y}$, so in particular $x \notin C_{y}$, a contradiction. Thus $x$ and $y$ must have different $Q$-values, and it follows that $Q$ is very essential over $P$.

Of course, $Q$ need not be a $p l$-cone; but by Theorem (2.6) we can extend $Q$ to 
a very essential $p l$-extension $Q^{\prime}$ of $P$. So applying Zorn's lemma, we get the existence of maximal very essential $p l$-extensions.

(2.7.1) Corollary. Let $(G, P)$ be a finite valued, divisible pl-group. Then every maximal very essential pl-extension is totally ordered.

Proof. According to (2.7) maximal very essential pl-extensions exist. But then by (2.5.1) they must be totally ordered. (The relation "very essential $p l$-extension of" is transitive; one can get it out of (2.4.1) without any trouble.)

The next result shows we can dispense with divisibility in the above corollary.

(2.8) TheOrem. Let $(G, P)$ be a pl-group and $\tilde{G}$ be its divisible closure; let $\widetilde{P}=\{x \in \widetilde{G}: n x \in P$, for some positive integer $n\}$. Then

(1) $(\widetilde{G}, \widetilde{P})$ is a pl-group; $(G, P)$ is a pl-subgroup.

(2) The map $C \mapsto \widetilde{C}=\{$ div. closure of $C$ in $\widetilde{G}\}$ is a 1-1 map of the set of o-ideals of $(G, P)$ onto the set of o-ideals of $(\tilde{G}, \widetilde{P}) . D \mapsto D \cap G$ is the inverse map; both maps preserve order.

(3) There is a 1-1 correspondence between the set of pl-extensions of $P$ and the set of pl-extensions of $\widetilde{P}$ in which $G$ is a pl-subgroup. The correspondence is given by $Q \leftrightarrow \tilde{Q}$, where $Q$ is a pl-extension of $P$, and $\widetilde{Q}=\{x \in \tilde{G}: n x \in Q$, for some positive integer $n$. The map preserves order in both directions.

(4) $Q$ is essential (resp. very essential) over $P$ if and only if $\widetilde{Q}$ is essential (resp. very essential) over $\tilde{P}$.

(5) $(G, Q)$ is finite valued if and only if $(\tilde{G}, \widetilde{Q})$ is finite valued. In fact, $x \in G \mid 0$ has as many values in $(G, Q)$ as it does in $(\widetilde{G}, \widetilde{Q})$.

Proof. (1) and (2) are well known (see [7]); the rest is straightforward.

(2.8.1) Corollary. Let $(G, P)$ be a finite valued pl-group. Then every maximal very essential pl-extension is totally ordered.

The proof of the following theorem is similar to that of (1.12) and will therefore be omitted. A different proof may be found in [11].

(2.9) TheOREM. Let $(G, P)$ be a finite valued pl-group, and $Q$ be a very essential Rz-extension; the map $\eta: \mathscr{R}(Q) \rightarrow \mathscr{R}(P)$ defined by $C=D \eta$ if and only if, given $x \in \bar{D} \backslash D, C$ is the unique $P$-value of $x$ that contains $D$, is a 1-1 map onto $\mathscr{R}(P)$, whose inverse $\rho: \mathscr{R}(P) \rightarrow \mathscr{R}(Q)$ preserves order. If $C=D \eta$ then the map $\theta_{D}: \bar{D} / D \rightarrow \bar{C} / C$ defined by $(D+y) \theta_{D}=C+y$ is an o-isomorphism onto $\bar{C} / C$. With $C \in \mathscr{R}(P), D=C \rho$ if and only if whenever $a$ is $P$-special at $C$ then $D$ is its $Q$-value.

Remark. With $(G, P)$ as in the theorem, and $Q$ an essential Rz-extension of $P$, we can still define $\rho: \mathscr{R}(P) \rightarrow \mathscr{R}(Q)$ as above. However, without the stronger assumption of the theorem $\rho$ is just an onto map that preserves order strictly; that is, $C \subset C^{\prime}$ implies that $C \rho \subset C^{\prime} \rho\left(C, C^{\prime} \in \mathscr{R}(P)\right)$.

Roughly speaking then, a very essential extension of a pl-group induces a 
shuffling of the regular o-ideals, preserving isomorphic factor groups. The next result shows that in certain cases this is characteristic of very essential extensions.

(2.10) THEOREM. Let $(G, P)$ be a finite valued, divisible pl-group, and suppose $\mathscr{R}(P)$ is the union of finitely many maximal chains. The following are then equivalent for a pl-extension $Q$ of $P$.

(i) $Q$ is very essential over $P$.

(ii) Considering $(G, P)$ as a pl-subgroup of $V=V\left(\mathscr{R}(P), R_{C}\right)$ as explained in Proposition (2.5), there is an extension $\tilde{\mathscr{R}}$ of the inclusion order of $\mathscr{R}(P)$, such that $V=V\left(\tilde{\mathscr{R}}, R_{C}\right)$ and $Q=G \cap V\left(\tilde{\mathscr{R}}, R_{C}\right)^{+}$.

Proof. That (ii) implies (i) is a consequence of (2.5). So all we must do is show that (i) implies (ii). Consider the map $\eta$ of Theorem (2.9); Let $\tilde{\mathscr{R}}$ be the order on $\mathscr{R}(P)$ induced from $\mathscr{R}(Q)$ by $\eta$. It is obvious that $\tilde{R}$ extends the inclusion order. Since $\mathscr{R}(P)$ can be covered by finitely many maximal chains we get that $V=V\left(\tilde{\mathscr{R}}, R_{C}\right)$ (notice that every set of $p$-disjoint elements in $V\left(\mathscr{R}(P), R_{C}\right)$ is finite). Also $Q=V\left(\tilde{\mathscr{R}}, R_{C}\right)^{+} \cap G$; for $x \in Q$ if and only if $D+x>D$ (rel. $Q$ ) for each $Q$-value $D$ of $x$, if and only if $C+x>C$ (rel. $P$ ) for each $P$-value $C$ of $x$ that contains a $Q$-value of $x$, if and only if every maximal component of $x$ relative to $\tilde{\mathscr{R}}$ is positive.

(2.11) Proposition. Let $(G, P)$ be a finite valued pl-group; then $P$ is the meet of its totally ordered very essential extensions.

Proof. By (2.8) we may assume, without loss of generality, that $G$ is divisible. Let $\left\{Q_{i}: i \in I\right\}$ be the set of totally ordered very essential extensions of $P$; of course $P \subseteq \bigcap\left\{Q_{i}: i \in I\right\}$. So suppose $x \notin P$; let $x=x_{1}+\cdots+x_{k}$ be a decomposition of $x$ into $P$-special components; we may take $x_{1}<0$. By the proof of Corollary (2.5.1) there is a very essential $p l$-extension $P_{1}$ of $P$ in which $\left|x_{2}\right| \ll-x_{1}$. By induction, there is a very essential $p l$-extension $P_{m}$ of $P_{m-1}$ in which $\left|x_{m+1}\right| \ll\left|x_{m}\right|, 1<m<k$. We therefore obtain a chain $P_{1}, \ldots, P_{k-1}$ of very essential $p l$-extensions of $P$, and in $P_{k-1}$ we have

$$
-x_{1} \gg\left|x_{2}\right| \gg \cdots \gg\left|x_{k}\right| \text {. }
$$

Letting $Q$ be a totally ordered, very essential extension of $P_{k-1}$ and hence of $P$, we see that $x \in-Q$, which implies that $x \notin\left\{Q_{i}: i \in I\right\}$. Thus the proof of Proposition (2.11) is complete.

(2.12) Proposition. Let $(G, P)$ be a pl-group such that $\mathscr{R}(P)$ has finitely many maximal chains. Then $P=\bigcap\left\{Q_{i}: 1 \leqq i \leqq m\right\}$, where each $Q_{i}$ is a totally ordered very essential extension of $P$. Conversely, if $(G, P)$ is a pl-group and $P$ is the meet of a finite number of total orders, then the number of elements in any set of p-disjoint elements is bounded by some finite cardinal.

Proof. By (2.8) we may assume $(G, P)$ is divisible. By a result of Conrad and 
Teller [3, Theorem 5.2] $(G, P) \cong V\left(\Lambda, H_{\lambda}\right)$, where $\Lambda$ is a finite partially ordered set, and for each $\lambda \in \Lambda, H_{\lambda}$ is an o-group. Let $\Lambda_{1}, \Lambda_{2}, \ldots, \Lambda_{m}$ be the total orders on $\Lambda$ which extend the given one, and let $Q_{i}=V\left(\Lambda_{i}, H_{\lambda}\right)^{+}, 1 \leqq i \leqq m$. (We remark that since $\Lambda$ is finite we have that $V\left(\Lambda, H_{\lambda}\right)=V\left(\Lambda_{i}, H_{\lambda}\right)=\Pi\left(\Lambda, H_{\lambda}\right)$.) Each $Q_{i}$ is clearly a very essential extension of $P$, and a total order. Suppose now that $x$ is $Q_{i}$-positive for each $1 \leqq i \leqq m$, and let $\lambda_{1}, \lambda_{2}, \ldots, \lambda_{n}$ be the maximal components of $x$ relative to $\Lambda$. For each $j(1 \leqq j \leqq n)$ there exists an $i=1,2, \ldots, m$, such that $\lambda_{j}>\lambda_{j^{\prime}}\left(\right.$ rel. $\left.\Lambda_{i}\right)$ for all $j^{\prime} \neq j$, which clearly implies that $x_{\lambda_{j}}>0$. Thus all the maximal components of $x$ relative to $\Lambda$ are positive, and so $x \in P$. This proves that $P=\bigcap\left\{Q_{i}: 1 \leqq i \leqq m\right\}$.

Conversely, suppose $P=\bigcap\left\{Q_{i}: 1 \leqq i \leqq m\right\}$ with each $Q_{i}$ totally ordered. Suppose there are $m+1 p$-disjoint elements $x_{1}, \ldots, x_{m}, x_{m+1}$ in $(G, P)$; choose $i=1, \ldots, m$; relative to $Q_{i}$ we must have $x_{1 \xi}<\cdots<x_{m \xi}<x_{(m+1) \xi}$, for some permutation $\xi$ in $S_{m+1}$. Thus each $Q_{i}$ induces a permutation of $S_{m+1}$, say $\xi_{i}$. Choose $k=1, \ldots, m+1$ such that $k \neq(m+1) \xi_{i}$, for any $i=1, \ldots, m$; let $x=x_{1}+\cdots+x_{k-1}-x_{k}+x_{k+1}+\cdots$ $+x_{m+1}$. Then $x \notin P$, but the above shows that $x \in Q_{i}$ for each $1 \leqq i \leqq m$, and this is a contradiction. Thus every set of $p$-disjoint elements has at most $m$ elements.

(2.12.1) Corollary. An l-group $(G, P)$ has finite basis if and only if $P$ is the meet of a finite number of total orders. If the number of total orders is $m$, then a basis for $(G, P)$ has at most $m$ elements.

The above corollary suggests that there may be ways to characterize properties of $l$-groups (such as having a basis, satisfying property $(F)$, being completely distributive, etc.) by looking at a family of total (very essential) extensions of the cone, which intersect in that cone.

Let $(G, P)$ be a $p l$-group; a very essential $l$-extension $Q$ of $P$ is minimal if there is no very essential $l$-extension of $P$ which $Q$ extends properly. We are going to prove the existence of minimal very essential $l$-extensions of finite valued $p l$-groups. We precede this result by some technical preliminaries.

(2.13) LEMMA. Let $(G, P)$ be a pl-group and $\left\{Q_{i}: i \in I\right\}$ be a chain of very essential l-extensions of $P$; let $Q=\bigcap\left\{Q_{i}: i \in I\right\}$, then for $x, y \in P, x \wedge_{Q} y=0$ if $x \wedge_{Q_{i}} \cdot y=0$, for some $i^{*} \in I$.

Proof. Of course $Q$ is a cone for a partial order on $G$. Suppose $a \leqq x, y$ (rel. $Q$ ); then $a \leqq x \wedge_{Q_{i}} y$, for all $i \in I$. In particular, $a \leqq x \wedge_{Q_{i^{*}}} y=0$ (rel. $Q_{i}$ ), for all $i \in I$ such that $Q_{i} \supseteq Q_{i^{*}}$. If $Q_{i} \subseteq Q_{i^{*}}$ then $x \wedge_{Q_{i}} y \leqq x \wedge_{Q_{i}} y=0$ (rel. $Q_{i^{*}}$ ), while $x \wedge_{Q_{i}} y \geqq 0$ (rel. $Q_{i}$ ); this implies that $x \wedge_{Q_{i}} y=0$ in this case also. Hence $a \leqq 0$ (rel. $Q_{i}$ ), for all $i \in I$, and so $a$ is $Q$-negative; this shows that $x \wedge_{Q} y=0$.

(2.14) Lemma. With $(G, P)$ a finite valued pl-group, and $\left\{Q_{i}: i \in I\right\}$ as in (2.13), let $Q=\bigcap\left\{Q_{i}: i \in I\right\}$. $Q$ is an l-cone; more precisely, for each $g \in G, g \vee_{Q} 0=g \vee_{Q_{i}} 0$, for all $Q_{i} \subseteq Q_{i^{*}}$, and some $i^{*} \in I$.

Proof. Let $a, b \in P$ be $P$-special elements, and suppose they are $p$-disjoint relative 
to $P$. Then $a \wedge_{Q} b=0$, or $a \ll b$, or $b \ll a$ (both rel. $Q$ ), depending upon whether $a \wedge_{Q_{i}} b=0$, for some $i \in I$, or $a \ll b$, or $b \ll a$, both relative to each $Q_{i}$.

Suppose then that $g \in G \backslash 0$, and let $g=g_{1}+\cdots+g_{n}$ be a decomposition of $g$ into $P$-special components. By a simple induction argument one easily sees that there is an $i_{0} \in I$ such that $\left|g_{j}\right| \ll\left|g_{k}\right|$ (rel. $Q$ ) if and only if $\left|g_{j}\right| \ll\left|g_{k}\right|$ (rel. $Q_{i_{0}}$ ); otherwise $\left|g_{j}\right| \wedge_{Q}\left|g_{k}\right|=0$ if and only if $\left|g_{j}\right| \wedge_{Q_{i_{0}}}\left|g_{k}\right|=0$. It also follows from the remark in the first paragraph that the above two equivalences hold for each $i \in I$ for which $Q_{i} \subseteq Q_{i_{0}}$. Since the $Q_{i}$ form a chain it is clear that $g \vee_{Q} 0=g \vee_{Q_{i}} 0$, for all $i \in I$, such that $Q_{i} \subseteq Q_{i_{0}}$. This proves the lemma.

(2.15) Theorem. Let $(G, P)$ be a finite valued pl-group. Minimal very essential l-extensions exist, and $P$ is the meet of such minimal extensions.

Proof. According to Corollary (2.8.1) $P$ has an $l$-extension. Of course if $P$ is an $l$-cone already there is nothing to prove. The existence of minimal very essential l-extensions then follows from Zorn's lemma and Lemma (2.14). Proposition (2.11) implies that $P$ is the meet of such extensions.

We know of one sufficient condition for an $l$-cone to be a minimal very essential extension of a given $p l$-cone. It is a corollary of the following result.

(2.16) Proposition. Let $(G, P)$ be a pl-group and $Q$ be an essential extension of $P$ such that $(G, Q)$ is an l-group. If $Q^{\prime}$ is a very essential l-extension of $P$ that contains $Q$, then $Q^{\prime}$ is very essential over $Q$.

Proof. Let $u$ and $v$ be $Q$-disjoint and suppose $u>v$ (rel. $Q^{\prime}$ ). Let $c$ and $d$ be $P$-positive parts of $u$ and $v$ respectively. Since $Q$ is essential over $P$, we have that $c \sim u$ and $d \sim v$ (rel. $Q$ ); in particular, $m c \geqq u$ and $n v \geqq d$ (rel. $Q$ ), for proper choices of positive integers. But $c$ and $d$ are $Q$-disjoint and hence so are $n m c$ and $d$. This implies (see [7, Theorem 4.5]) that $n m c$ and $d$ are in fact $P$-disjoint. On the other hand $n m c \geqq n u \geqq n v \geqq d$ (rel. $Q^{\prime}$ ); it follows that $n m c \gg d$, since $Q^{\prime}$ is very essential over $P$, and also $c \gg d$, which in turn implies that $u \gg v$, all relative to $Q^{\prime}$. This proves that $Q^{\prime}$ is very essential over $Q$.

(2.16.1) Corollary. Let $(G, P)$ be a pl-group, and $Q$ be a very essential l-extension of $P$ having the property that whenever $x$ and $y$ are disjoint relative to $P$, then they are $Q$-disjoint as well. Then $Q$ is minimal very essential over $P$.

Proof. Suppose $Q$ contains a very essential $l$-extension $Q^{\prime}$ of $P$ properly. Let $x$ and $y$ be $Q^{\prime}$-disjoint elements such that $x>y$ (rel. $Q$ ), and hence $x \gg y$ (rel. $Q$ ) by (2.16). Let $a$ and $b$ be $P$-positive parts of $x$ and $y$ respectively. Then since $a \sim x$ and $b \sim y$ (rel. $Q^{\prime}$ ), we have that $a$ and $b$ are $Q^{\prime}$-disjoint while $a \gg b$ (rel. $Q$ ). However, $a$ and $b$ must also be $P$-disjoint, and so by our standing hypothesis $a \wedge_{Q} b=0$, which amounts to a contradiction. Therefore $Q$ must be minimal and we are done.

The condition in (2.16.1) does not seem to be very satisfactory. For one the converse of $(2.16 .1)$ is false. Consider: 
(2-C) Let $\Lambda=\{1,2,3,4,5\}$ ordered as in the picture below.

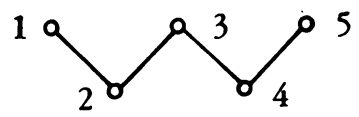

Let $(G, P)=V(\Lambda, R)=\Pi(\Lambda, R) ;(G, P)$ is a finite valued $p l$-group. Let $\Lambda^{\prime}=\Lambda$ with the following order on it:

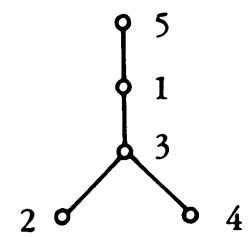

Then $\Lambda^{\prime}$ extends $\Lambda$; if we let $(G, Q)=V\left(\Lambda^{\prime}, R\right)$ then $Q$ is very essential over $P$ (in view of (2.10)). $Q$ is also minimal over $P$ (again by (2.10)); but letting $x=(1,0,0,0,0)$ and $y=(0,0,0,0,1)$ then $x \wedge_{P} y=0$, while $y \gg x$ (rel. $\left.Q\right)$.

In fact a $p l$-group may not have any minimal very essential $l$-extensions satisfying the condition in (2.16.1):

(2-D) Let $\Lambda=\{1,2,3,4,5\}$ ordered as in the following picture:

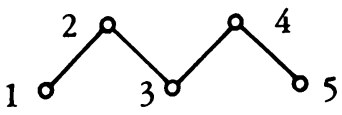

Let $(G, P)=V(\Lambda, R)=\Pi(\Lambda, R) ;(G, P)$ is a finite valued $p l$-group. Let $Q$ be a very essential $l$-extension of $P$; then $Q=V\left(\Lambda^{\prime}, R\right)^{+}$, for some order extension $\Lambda^{\prime}$ of $\Lambda$ which is a root system. This forces 2 to be comparable to 4 (rel. $\Lambda^{\prime}$ ), say $2<4$. Take $x=(1,0,0,0,0)$ and $y=(0,0,0,1,0)$; then $x$ and $y$ are $P$-disjoint, while $x \ll y$ (rel. $Q$ ), and so $Q$ over $P$ does not satisfy the condition of (2.16.1). In particular then, no minimal very essential $l$-extension had this property.

\section{BIBLIOGRAPHY}

1. R. D. Byrd and J. T. Lloyd, Closed subgroups and complete distributivity in lattice-ordered groups, Math. Z. 101 (1967), 123-130. MR 36 \#1371.

2. P. F. Conrad, J. Harvey and C. Holland, The Hahn embedding theorem for abelian latticeordered groups, Trans. Amer. Math. Soc. 108 (1963), 143-169. MR 27 \#1519.

3. P. F. Conrad and J. R. Teller, Abelian pseudo lattice-ordered groups (preprint).

4. P. F. Conrad, The structure of a lattice-ordered group with a finite number of disjoint elements, Michigan Math. J. 7 (1960), 171-180. MR 22 \#6854.

5. - Some structure theorems for lattice-ordered groups, Trans. Amer. Math. Soc. 99 (1961), 212-240. MR 22 \#12143.

6. - The lattice of all convex l-subgroups of a lattice-ordered group, Czechoslovak Math. J. 15 (90) (1965), 101-123. MR 30 \#3926.

7. - Representation of partially ordered abelian groups as groups of real valued functions, Acta Math. 116 (1966), 199-221. MR 34 \#1418. 
8. P. F. Conrad, Introduction à la théorie des groupes réticulés, Université de Paris, Faculté des Sciences de Paris, Secrétariat mathématique, Paris, 1967. MR 37 \#1289.

9. L. Fuchs, Teilweise geordnete algebraische Strukturen, Studia Math. Mathematische Lehrbücher, Band XIX, Vandenhoeck \& Ruprecht, Göttingen, 1966. MR 34 \#4386.

10. — Riesz vector spaces and Riesz algebras, Queen's Papers in Pure and Appl. Math., no. 1, Queen's University, Kingston, Ont., 1966. MR 34 \#3288.

11. J. Martinez, Dissertation, Tulane University, New Orleans, La., 1969.

12. J. R. Teller, On abelian pseudo lattice ordered groups, Pacific J. Math. 27 (1968), 411-419. MR 38 \#3203.

13. S. Wolfenstein, Valeurs normales dans un groupe réticulé, Atti Accad. Naz. Lincei Rend. Cl. Sci. Fis. Mat. Natur. (8) 44 (1968), 337-342. MR 38 \#3201.

University of Florida, Gainesville, Florida 32601 